\title{
Direct and apparent compensation in plant-herbivore interactions
}

\author{
A. Lebon ${ }^{\mathrm{a}}$, L. Mailleret ${ }^{\mathrm{b}, \mathrm{c}, \mathrm{d}, \mathrm{e}}$, Y. Dumont ${ }^{\mathrm{a}, *}, \mathrm{~F}$. Grognard ${ }^{\mathrm{e}}$ \\ ${ }^{a}$ CIRAD, Umr AMAP, Montpellier, France \\ ${ }^{b}$ INRA, UMR 1355 ISA, Teapea, Sophia Antipolis, France \\ ${ }^{c}$ Université Nice Sophia Antipolis, UMR 1355 ISA, Teapea, Sophia Antipolis, France \\ ${ }^{d}$ CNRS, UMR 7254 ISA, Teapea, Sophia Antipolis, France \\ ${ }^{e}$ BIOCORE, INRIA, Sophia-Antipolis, France
}

\section{Abstract}

The potential positive effects of herbivores on plants have been the subject of debates for decades. While traditionally, herbivory was considered to have a negative impact on plants, some studies also reported possible mutualism between plants and herbivores. Plant defences, and in particular tolerance and resistance, seem to play an important role in shaping plant-herbivore interactions. The aim of this study is to show how a direct plant compensation mechanism translates into apparent compensation, i.e. the long-term biomass response to herbivory, in simple plant-herbivore models. A special emphasis is then put on how it interacts with resistance mechanisms. A qualitative study of the proposed models shows that they can exhibit different plant-herbivore patterns, including neutral, antagonistic(negative apparent compensation) and mutualistic (positive apparent compensation) interactions. Moreover, it is shown that density dependence plays a crucial role since, for a given system, the realized plant-herbivore pattern critically depends on the initial plant and herbivore levels. Our study shows the importance of direct compensation for the presence of plant-herbivore mutualism, a finding which we show has significant implications both in ecosystems ecology and in agricultural pest management.

Keywords: Mathematical modelling, population ecology, plant-herbivore interactions, plant defences, grazing optimization hypothesis, agronomic application

${ }^{*}$ Corresponding author. Tel. +33467614908 ; fax. +33 467 615668. Address: CIRAD, TA A51/PS2 34398 Montpellier cedex 5, France. E-mail address: yves.dumont@cirad.fr. 


\section{Introduction}

It has long been considered that herbivory has a negative impact on plants, both in terms

of growth and reproduction. Numerous studies and an abundant literature about herbivory for almost all types of ecosystems support this view: herbivory not only can decrease leaf area, but also nutrient stocks, photosynthetic capacity (Zangerl et al., 2002), reproductive success (Quesada et al., 1995) or vegetative growth (Meyer, 1998). Yet, in response to herbivory, plants have evolved different defence strategies, which can be broadly categorized into resistance and tolerance (Strauss and Agrawal, 1999; Agrawal, 2000).

On the one hand, plant resistance relies on the production of defences dedicated to reduce herbivore performances or preferences (Agrawal, 2000). These defences, like mechanical structures (spines, hairs, leaf toughness, ...) or chemical defences (toxins, repellants, ...), may be constitutive or induced by herbivore attacks, but are ultimately costly for plants in terms of growth or reproduction ((Strauss et al., 2002) and references therein, (Arimura et al., 2005)).

On the other hand, plant tolerance aims to reduce the negative impact of herbivory on plant fitness and/or biomass once it has occurred. Plant tolerance is strongly linked with the compensation effect, a process by which plants may respond positively, in terms of growth and/or fitness, to herbivory (McNaughton, 1983; Trumble et al., 1993; Strauss and Agrawal, 1999). More precisely, the "herbivory (or grazing) optimization hypothesis" $(\mathrm{HOH})$ states that only low to moderate levels of herbivory can trigger a positive response in plants (Dyer, 1975; McNaughton, 1979; Hilbert et al., 1981; Paige and Whitham, 1986), see Figure 1. This principle, and more specifically plant compensation itself, has been the matter of a debate during the $80 \mathrm{~s}$ and $90 \mathrm{~s}$ and has even been dismissed by some authors (Belsky, 1986; Belsky et al., 1993). This is probably because the term "compensation" is by itself confusing (Brown and Allen, 1989). Indeed an increase in plant growth rate does not necessarily lead to an increase in the vegetative and/or reproductive biomass: plant biomass dynamics are primarily driven by their growth, but also by herbivore consumption. Thus, they may very well respond positively to herbivory (i.e. growth increases) while displaying 
decreasing biomass levels, simply because herbivore consumption is strong. Plant biomass is indeed the net result of a complex compensation feedback loop encompassing improved plant growth, the ensuing increased herbivore level, and the resulting amplification of herbivory, which may or may not lead to the observation of increased plant biomass. Hence, plant compensation needs to be considered at two levels:

- direct compensation, the basic positive response of plants to herbivores, which is measured in terms of growth or fitness;

- apparent compensation, the net result of the whole plant-herbivore interaction, which is measured as the overall effect of herbivores on plant biomass.

In this article, we will focus on plants which can express direct compensation and investigate apparent compensation. Sticking to the plant compensation terminology we will refer to over- and under-compensation as positive and negative instances of apparent compensation. These correspond to mutualistic and antagonistic plant-herbivore interactions in the more general ecological terminology.

Direct plant compensation can be brought about by different mechanisms, linked for instance to a release of bud dormancy, a modification of self-shading distribution, biomass reallocation or even increases in the rate of photosynthesis. Photosynthesis increases may occur because leaves often function below their maximum capacity (Paul and Foyer, 2001) and can be caused by carbon reallocation within plants following herbivory (Thomson et al., 2003). It can also simply be induced by a herbivore elicited increase of plants primary metabolism (Dyer et al., 1995; Poveda et al., 2010). These mechanisms have been observed on various wild or domesticated plants even for important herbivory levels (see Thomson et al. (2003) and references therein). For instance, the butterprint preserves its growth and reproduction after up to $75 \%$ defoliation (Mabry and Wayne, 1997). Wild radish plants behave similarly after up to $25 \%$ defoliation (Agrawal, 2000). Such effects have also been reported for agronomic plants, as cotton (Sadras, 1996), tomato (Trumble et al., 1993) or cucumber (Thomson et al., 2003), and Poveda et al. (2010) even showed that the Guatemalan potato moth does increase potato plants productivity. Other causes of direct compensation 
are linked to the functioning of ecosystems themselves. For instance, herbivores can modulate the competition between plants for light, nutrients or water, inducing increases in the overall ecosystem primary productivity (Crawley, 1989; Center et al., 2005). However, the main ecological driver of direct plant compensation is soil fertilization by herbivore dejections and the related increase in nutrients turnover (DeAngelis, 1992).

The processes undelying direct compensation are thus very diverse. They have been recorded on various plant-herbivore systems, such as on grasses-mammalian browsers (McNaughton, 1979; Paige and Whitham, 1986), agronomic plants or grasses-birds (Dyer, 1975; Hik and Jefferies, 1990), and plants-insects (Williamson et al., 1989; Dyer et al., 1993; Poveda et al., 2010). Yet, whether and how direct compensation translates into apparent over- or under-compensation needs to be addressed. Classical examples of plants overcompensating are scarlet Gilia and field gentians, which can double their fitness as compared to undamaged plants (Paige and Whitham, 1986; Lennartsson et al., 1998). Agronomic plants also exhibit overcompensation: corn crops tend to produce more grain when fed upon by Red-Winged Blackbirds (Dyer, 1975) and cotton crops appear to yield more in presence of cotton boll worms (Dyer et al., 1993). Another instance of the phenomenon concerns the aforementioned potato plants, which can double their biomass when confronted to small densities of larvae of the Guatemalan potato moth but see it halved when the infestation is severe (Poveda et al., 2010). Hence, the mechanism can be density dependent. The process by which direct compensation translates into apparent over-compensation is thus complex; understanding it better is an important question, which pertains to both the ecological and agronomic sciences.

To gain further insights on apparent plant compensation, we developed a dynamical plant-herbivore model. To our knowledge, most such models predict that plant-herbivore systems can only display undercompensation at equilibrium (Loreau, 1995; de Mazancourt et al., 1998; Ryrie and Prentice, 2011; Fukui et al., 2012), but see (de Mazancourt, 2001) for an exception. In this study, we considered a simple two-dimensional plant-herbivore model with built-in direct plant compensation. We modelled the response of plants to herbivory 
through a direct dependency of the plant compartment growth rate on the number of herbivores (Fig. 1). In a first step, we concentrated on the compensation mechanism ( Direct compensation" case). We showed that, depending on two threshold quantities inferred from the analysis, stable plant-herbivore co-existence equilibria may be characterized by either under- or over-compensation by plants. Overcompensation situations are strongly density dependent so that overcompensation equilibria may be reached only if the initial herbivore population is large enough. Since both compensation and resistance may be jointly expressed by plants (Nunez-Farfan et al., 2007), in a second step we incorporated plant resistance in the model, considering that the herbivore growth rate is a non-monotonic function of the plant compartment level ("Direct compensation and Resistance" case). The dynamical patterns produced by the model when both mechanisms are taken into account were much richer. As a special point of interest, the "Direct compensation and Resistance" model can exhibit, for a given set of parameters, over- and under-compensation co-existence equilibria being both stable. Again, the long-term model behaviour was density dependent: yet, in this situation, overcompensation equilibria were reached from low herbivore levels, while undercompensation ones are consequences of high herbivore levels.

This paper is organized as follows. In section 2, we present the general plant-herbivore model and state the specific hypotheses of the "Direct compensation" and "Direct compensation and Resistance" models. Section 3 is dedicated to the mathematical analysis. Results are illustrated through simulations and bifurcation diagrams worked out on particular functional forms of the models in section 4. A discussion concludes this contribution, with special emphasis on the agronomic consequences of overcompensation by plants and how they may influence the design of agricultural pest management programs. 


\section{Materials and methods}

\subsection{Modelling Plant-Herbivore Dynamics}

The model consists of two coupled ordinary differential equations, describing the temporal evolution of plant biomass $B$, and the herbivore (or pest) population $H$. It is written as :

$$
\left\{\begin{array}{l}
\dot{B}=r_{B}(H) B-\delta(B) B-\phi(B) B H \\
\dot{H}=\alpha(B) H-\mu H
\end{array}\right.
$$

with $B, H$ taking values in $\mathbb{R}^{+}, B(0)>0$, and $H(0) \geq 0$. The modelling hypotheses are described in the following sections.

\subsubsection{Processes without herbivores}

In the absence of herbivores, the dynamics of the plant biomass is the net result of the biomass production through growth and reproduction (stemming from photosynthesis, ...) and the negative effect on the biomass of maintenance or intraspecific competition. The biomass production per unit of time is assumed to be a linear function of the available biomass, i.e $r_{B}(0) B$ where $r_{B}(0)>0$ is the biomass production rate. The loss of biomass, per unit of time, is represented by the function $\delta(B) B$ (Marcelis et al., 1998). We assume that $\delta(B)$ is a positive increasing function of the biomass (Lebon et al., 2012; Reich et al., 2006; Noy-Meir, 1975). Note that we may have $\delta(0) \geq r_{B}(0)$ so that the plant biomass decays even without herbivory. Such a situation can occur under stress conditions, like lack of nutrients, water deficit, or/and soil salinity. Since we do not take into account other environmental perturbations than herbivory, we assume that $\delta(0)<r_{B}(0)$ for the rest of the paper.

In the absence of plants, the herbivore population cannot grow $(\alpha(0)=0)$ and decays at a constant rate $\mu$.

\subsubsection{Plants-herbivores interactions}

As described in the introduction, herbivores and plants may maintain complex interactions, through biomass consumption/herbivore growth, and plant tolerance and resistance mechanisms. 
Tolerance, which in the present study is represented by direct compensation, may impact different plant features leading to various responses, that are often related to plant growth or reproduction. Thus, direct compensation is more or less linked to the biomass dynamics of damaged plants, and here, is taken into account through $r_{B}(H)$, the biomass production rate. As long as $H=0$, the plant biomass grows "normally", driven by $r_{B}(0)$ and $\delta(B)$. However, the herbivory-dependent compensation capacity is immediately triggered at the moment of herbivores invasion: an increase of plant growth occurs in order to limit herbivory damage. When herbivory disappears, normal growth immediately takes place again. Thus, to model direct compensation, we will suppose that $r_{B}(H)$ is increasing for small $H$ and concave up to $H=H_{m}$ where $r\left(H_{m}\right)=r(0)$, which essentially mimics the herbivory optimization curve of Figure 1. Concavity is imposed because it is assumed that direct compensation cannot be an accelerating function of $H$, the smallest herbivore sensing immediately triggering an increase in plant growth. Upper-boundedness is obviously required to prevent an unrealistic infinite growth rate. Note that the present study can handle positive $H_{m}$ values, but also $H_{m}=0$, which means that the compensation effort is outweighed by the negative direct effect of the pest on the metabolism, or $H_{m}=+\infty$, which corresponds to an ever increasing compensation. It is not constrained to the non-monotonicity of the $\mathrm{HOH}$.

Standard Consumer-Resource (Plant-Herbivore) interactions are usually modelled through a functional response (here $\phi(B) B$ ), and a numerical response (here $\alpha(B)$ ). Various choices are possible for the functional response, like for instance, the different biomass densitydependent functions proposed by Holling (Holling, 1959). Usually, the numerical response also depends on the available biomass and is classically taken to be proportional to $\phi(B) B$, for mass balance considerations. Releasing that constraint can help to represent various defence mechanisms. In order to stay as generic as possible, we will just impose that $\phi(B)$ is a positive, non-increasing function defined for all $B \in \mathbb{R}^{+}$. Such hypotheses encompass classical monotonic functional responses as the Holling II, as well as unimodal functional responses as the Holling IV.

When the only considered defence is direct compensation, the numerical response, $\alpha(B)$, 
is chosen as an increasing, function, with $\alpha(0)=0$. It would of course not be relevant to consider $\mu>\lim _{B \rightarrow+\infty} \alpha(B)$, when $\alpha(B)$ is upper-bounded, since herbivore mortality would always be higher than herbivore growth rate, and the herbivore population would decay until extinction. For the rest of the paper, we then assume that $\mu<\lim _{B \rightarrow+\infty} \alpha(B)$. In the "Direct compensation" case, $\alpha(B)=\mu$ has a single solution that we will denote $B^{*}$.

When direct compensation and resistance are both considered, functional and numerical responses can be more complex. Indeed resistance mechanisms can prevent/limit biomass consumption and impede the herbivore growth rate. Resistance defences can take various forms, like the release of volatile compounds (Arimura et al., 2005; Heil, 2010; Gibson and Pickett, 1983), and toxins, or mechanical structures, like tissue hardness, pubescence, sclerophylly ...(Hanley et al., 2000; Fernandes, 1994), which limit consumption, hence herbivore growth; both consumption and growth then increase for small biomasses and then decrease for $B$ large since, as plants grow and age, mechanisms such as tissue hardness become more efficient. Such non-monotonic functional and numerical responses can be found in (Li et al., 2006; Feng et al., 2008, 2011), where the ingestion of toxins reduces herbivory or influences its plant selectivity. Additional resistance defences include digestibility reduction, or poisoning of the herbivore through toxins. They can be modelled through non-monotonic numerical responses. In the spirit of $\mathrm{Li}$ et al. (2006), we assume that when herbivores consume too much poisonous biomass, $\alpha(B)$ decreases. The growth rate is then hampered as a function of the biomass consumption rate, $\phi(B) B$. It could even lead to increased mortality beyond some point. Thus, we define

$$
\alpha(B)=\phi(B) B \times \psi(\phi(B) B)
$$

with $\psi$ a decreasing function of its argument. For instance, assuming $\phi(B) B=\frac{\phi_{0} B}{1+q_{0} B}$, a poisoning function of the form $\psi(x)=\frac{p_{0}}{1+\pi x}$ simply yields a reduced (but still increasing) growth rate, while $\psi(x)=\frac{p_{0}}{(1+\pi x)^{2}}$ results in a function in the form $\frac{\rho B^{2}+\eta B}{B^{2}+\nu B+\theta}$ which, provided $\rho \nu-\eta>0$, is increasing and then decreasing towards $\rho$.

Summarizing the previous explanations, $\alpha(B)$ verifies $\alpha(0)=0$ and $\alpha^{\prime}(B)>0$ (resp. $<$ ) for $B<B_{m}$ (resp. $>$ ). A typical choice for $\alpha$ would be $\alpha(B)=\frac{\eta B}{B^{2}+\nu B+\theta}$ which is 
classically used to model biomass poisoning/inhibition by substrate in bioreactors (Andrews, 1968), some enzymatic kinetics (Haldane, 1930), or group defence by prey against predators (Freedman and Wolkowicz, 1986). Li et al. (2006) proposed a function of the form $\alpha(B)=$

$\phi(B) B\left(1-\frac{\phi(B) B}{G}\right)$, which also satisfies our hypotheses on $\alpha$ for $\phi(B) B$ of Holling type I or II. Finally, assuming $\mu<\alpha\left(B_{m}\right)$ implies that $\alpha(B)$ has one or two solutions $B_{1}^{*}$ and $B_{2}^{*}$ where $\alpha^{\prime}\left(B_{1}^{*}\right)>0$ and $\alpha^{\prime}\left(B_{2}^{*}\right)<0$, and $B_{1}^{*}<B_{m}<B_{2}^{*}$. However, if $\mu<\lim _{B \rightarrow+\infty} \alpha(B)<\alpha\left(B_{m}\right)$, there will be only one solution of $\alpha(B)-\mu=0$.

Table 1 summarizes all the hypotheses on the aforementioned functions.

\section{Results}

In both the "Direct compensation" and "Direct compensation and resistance " cases, according to the previous hypotheses, it can be shown that the solutions stay bounded and in the non-negative orthant $\mathbb{R}_{+}^{2}$, if the initial conditions are non-negative.

\subsection{Direct compensation}

\subsubsection{Existence of equilibria}

Looking for the equilibria in system (1) is equivalent to solving the following system:

$$
\begin{cases}B\left[r_{B}(H)-\delta(B)-\phi(B) H\right] & =0, \\ H[\alpha(B)-\mu] & =0\end{cases}
$$

When there is no herbivore, i.e. $H^{*}=0, B$ needs to satisfy $B\left[r_{B}(0)-\delta(B)\right]=0$, so that, since $\delta(0)<r_{B}(0)$, two equilibria are possible: one trivial equilibrium $(B, H)=(0,0)$ and one herbivore-free equilibrium $(B, H)=\left(B_{\sharp}, 0\right)$, where the assumptions on $\delta$ allow for the definition of $B_{\sharp}=\delta^{-1}\left(r_{B}(0)\right)$.

Otherwise, when $H^{*}>0$ and since $\alpha$ is increasing, $\alpha(B)=\mu$ yields a unique solution $B^{*}$. Finally, $B^{*}$ being fixed, we deduce $H^{*}$ from the following equation :

$$
r_{B}(H)=\delta\left(B^{*}\right)+\phi\left(B^{*}\right) H
$$

However, since we have not specified most functions, no analytical solution of equation (3) can be obtained. Yet, the number of $H^{*}$ solutions can be identified since the shapes 
of the functions of $H$ on both sides of the equality are known: function $r_{B}(H)$, with the hypotheses given in Table 1 on the left, and an increasing linear function on the right. The different situations are illustrated on Figure 2 where both sides of equation (3) are represented and where we can identify the most important parameters for the number of equilibria. The situations are first segregated on the basis of the relative values of the functions at $H=0: r_{B}(0)$ and $\delta\left(B^{*}\right)$. If $r_{B}(0)>\delta\left(B^{*}\right)$, equation (3) has a unique solution, that we will denote $H_{2}^{*}$; note that, since $r_{B}(0)=\delta\left(B_{\sharp}\right)$ and $\delta$ is increasing, this situation occurs when $B_{\sharp}>B^{*}$, hence an undercompensation case. This leads us to the definition of an apparent compensation index

$$
\mathcal{C}_{a}=\frac{B^{*}}{B_{\sharp}}
$$

$\mathcal{C}_{a}$ is larger than 1 in cases of overcompensation and smaller than 1 for undercompensation. The latter is illustrated on Figure 2(i) with $r_{B}(0)=\delta\left(B_{\sharp}\right)$ and $\delta\left(B^{*}\right)=\delta\left(\mathcal{C}_{a} B_{\sharp}\right)$ the values of both sides of equation (3) at $H=0$. If $r_{B}(0)<\delta\left(B^{*}\right)$, more situations can be expected as can be seen on Figures 2(ii) and (iii), which present two and zero equilibria. These are potential overcompensation situations since they correspond to $\mathcal{C}_{a}>1$. Note that, despite $\mathcal{C}_{a}$ being larger than 1, there is no effective overcompensation in case (ii) since no corresponding equilibrium exists.

More precisely, we can identify a sufficient condition to discriminate between the cases with two and zero equilibria when $\mathcal{C}_{a}>1$ (see Fig. 2(ii)-(iii)). Namely, if the slope of $\delta\left(B^{*}\right)+\phi\left(B^{*}\right) H$ is larger than that of $r_{B}(H)$ in $H=0$, the linearity of the first one and concavity of the second one in $H$ prevent the existence of an intersection. This can be written as:

$$
\phi\left(B^{*}\right)>r_{B}^{\prime}(0)
$$

This leads us to the definition of a short-term compensation index

$$
\mathcal{C}_{s}=\frac{r_{B}^{\prime}(0)}{\phi\left(B^{*}\right)}
$$

We then conclude that, if $\mathcal{C}_{s}<1$ and $\mathcal{C}_{a}>1$, equation (3) has no solution. In fact, $\mathcal{C}_{s}$ is a negative index of overcompensation: if $\mathcal{C}_{s}<1$, the system will never reach a better biomass 
than $B_{\sharp}$. On the contrary, if $\mathcal{C}_{s}>1$ and $\mathcal{C}_{a}>1$, the system may reach an overcompensation equilibrium (depending on the initial conditions and the parameters). However, the situation of Fig. 2(ii) could both occur with $\mathcal{C}_{s}<1$ (illustrated) or $\mathcal{C}_{s}>1$.

The qualitative analysis of equation (3) thus yields the following proposition about the existence of positive equilibria, which depends on $\mathcal{C}_{s}$ and $\mathcal{C}_{a}$, the compensation indices.

Proposition 1. With the functions defined as in Table 1 and $\alpha(B)$ satisfying the "Direct compensation" assumptions,

- when $\mathcal{C}_{a}<1$, system (1) has one positive equilibrium (see Fig. 2(i))

- when $\mathcal{C}_{a} \geq 1$ and $\mathcal{C}_{s}<1$ system (1) has zero positive equilibrium (see Fig. 2(ii))

- when $\mathcal{C}_{a} \geq 1$ and $\mathcal{C}_{s} \geq 1$, system (1) has zero or two positive equilibria (see Fig. 2(ii) and (iii)).

All situations are not covered in Proposition 1: in the third case, tangency between both curves could limit the intersection to one value of $H^{*}$; also both curves could be identical over an interval. However, any small variation of one parameter would destroy such a situation and yield the third case of Proposition 1. We will not comment any further on these non robust cases.

If $\mathcal{C}_{a}<1$ (see Fig. 2(i)), the plant equilibrium biomass without herbivores is higher than the one with: it is a case of under-compensation, $\left(B^{*}, H_{2}^{*}\right)$. Assuming $\mathcal{C}_{a}>1$, when (3) has two solutions (see Fig. 2(iii)), then the plant equilibrium biomass with herbivores is higher than the plant equilibrium biomass without herbivores: there generically are two cases of overcompensation $\left(\left(B^{*}, H_{1}^{*}\right)\right.$ and $\left.\left(B^{*}, H_{2}^{*}\right)\right)$. If (3) has no solution (see Fig. 2(ii)), no compensation equilibrium exists despite $\mathcal{C}_{a}>1$.

Though it looked to be strictly built for the analysis of Figure 2, the short-term compensation index terminology for $\mathcal{C}_{s}$ can be justified by considering the reaction of the plant biomass to the invasion of a small herbivore population. In the case where $\mathcal{C}_{a}>1$, which is the only situation in which $\mathcal{C}_{s}$ matters as seen in Proposition 1, we study the invasion of a small population of herbivores in a system at equilibrium $\left(B_{\sharp}, 0\right)$. We check if it can induce an increase of the plant biomass through direct plant compensation weighted down 
by herbivore consumption:

$$
\frac{\dot{B}\left(B_{\sharp}, H\right)}{B_{\sharp}}=r_{B}(H)-\delta\left(B_{\sharp}\right)-\phi\left(B_{\sharp}\right) H \approx r_{B}(0)+r_{B}^{\prime}(0) H-\delta\left(B_{\sharp}\right)-\phi\left(B_{\sharp}\right) H .
$$

Since $r_{B}(0)=\delta\left(B_{\sharp}\right)$ and $\phi\left(B^{*}\right)<\phi\left(B_{\sharp}\right)$, this implies that

$$
\frac{\dot{B}\left(B_{\sharp}, H\right)}{B_{\sharp}}<\left(r_{B}^{\prime}(0)-\phi\left(B^{*}\right)\right) H=\phi\left(B^{*}\right)\left(\mathcal{C}_{s}-1\right) H .
$$

Condition $\mathcal{C}_{s}>1$ is then necessary for the instantaneous compensation due to a small herbivore invasion to induce a direct increase of the biomass from its herbivore-free equilibrium; hence the short term compensation definition. Contrariwise $\mathcal{C}_{s}<1$ precludes plant overcompensation. If $B_{\sharp}=0$, such an interpretation does not make sense since the invasion of herbivores would not change the biomass, which stays 0 .

\subsubsection{Local stability analysis}

Local asymptotic stability or instability of each equilibrium is studied by computing the eigenvalues of the Jacobian Matrix $\mathcal{J}(X)$ related to system (1) and is detailed in AppendixA. It yields:

Proposition 2. For system (1) with the functions defined as in Table 1 and $\alpha(B)$ satisfying the "Direct compensation" assumptions,

- $\left(B_{\sharp}, 0\right)$ is locally asymptotically stable if $\mathcal{C}_{a}>1$;

- $\left(B^{*}, H_{1}^{*}\right)$ is always unstable when it exists;

- $\left(B^{*}, H_{2}^{*}\right)$ is asymptotically stable when it exists if $\delta^{\prime}\left(B^{*}\right)+\phi^{\prime}\left(B^{*}\right) H_{2}^{*}>0$.

For all concerned equilibria, stability is lost if the corresponding inequalities are strictly reversed.

The stability situation is summarized in Fig.2 and Table 2. In the figure, the small circles correspond to the $\left(B_{\sharp}, 0\right)$ and $\left(B^{*}, H_{j}^{*}\right)$ equilibrium points (even though only the $H$ coordinate is read on the figure), with the white circles corresponding to unstable equilibria, the black ones to stable equilibria and the gray ones to equilibria whose stability depends on an additional condition given in Proposition 2. When the latter condition is not verified, $\left(B^{*}, H_{2}^{*}\right)$ loses its stability through a Hopf bifurcation. The $j$ indices labelling the equilibria represent the herbivore level, i.e. $H_{1}^{*}$ corresponds to a small level while $H_{2}^{*}$ to a high one. The instability of $(0,0)$ is not illustrated. 


\subsection{Direct compensation and Resistance}

When $\alpha(B)$ is non-monotonic, the equilibria still need to satisfy (2) so the analysis does not change much. The only difference is initiated in the solutions of $H[\alpha(B)-\mu]=0$, which yields $H=0$ as earlier, but also one or two positive solutions: $B_{1}^{*}$ and possibly $B_{2}^{*}$ with $B_{1}^{*}<B_{m}<B_{2}^{*}$, where the maximum of $\alpha\left(\right.$.) is reached in $B=B_{m}$. Positive equilibria can then be built on $B_{1}^{*}$ and $B_{2}^{*}:\left(B_{1}^{*}, H_{11}^{*}\right),\left(B_{1}^{*}, H_{12}^{*}\right),\left(B_{2}^{*}, H_{21}^{*}\right)$, and $\left(B_{2}^{*}, H_{22}^{*}\right)$. The analysis of existence of the $H_{i j}^{*}$ is done exactly as in Section 3.1, once for $B_{1}^{*}$ and once for $B_{2}^{*}$. This imposes the definition of two apparent and two short term compensation indices:

$$
\begin{gathered}
\mathcal{C}_{a 1}=\frac{B_{1}^{*}}{B_{\sharp}} \quad \text { and } \quad \mathcal{C}_{a 2}=\frac{B_{2}^{*}}{B_{\sharp}} \\
\mathcal{C}_{s 1}=\frac{r_{B}^{\prime}(0)}{\phi\left(B_{1}^{*}\right)} \quad \text { and } \quad \mathcal{C}_{s 2}=\frac{r_{B}^{\prime}(0)}{\phi\left(B_{2}^{*}\right)}
\end{gathered}
$$

with $\mathcal{C}_{a 2}>\mathcal{C}_{a 1}$ and $\mathcal{C}_{s 2} \geq \mathcal{C}_{s 1}$ since $B_{2}^{*}>B_{1}^{*}$ and $\phi$ is non-increasing. Proposition 1 then still holds with the caveat that it must be applied twice, once with $B_{1}^{*}, \mathcal{C}_{a 1}$, and $\mathcal{C}_{s 1}$, and once with $B_{2}^{*}, \mathcal{C}_{a 2}$, and $\mathcal{C}_{s 2}$, yielding between 0 and 4 positive equilibria for system (1).

The stability analysis which is essentially similar to the previous one is presented in AppendixB; results are summarized in the following Proposition.

Proposition 3. For system (1) with $r_{B}(H), \delta(B), \phi(B)$ and $\alpha(B)$ defined as in Table 1 and $\alpha(B)$ satisfying the "Direct compensation and Resistance" assumptions,

- $\left(B_{\sharp}, 0\right)$ is asymptotically stable if $\alpha\left(B_{\sharp}\right)<\mu$, that is if $B_{\sharp}<B_{1}^{*}<B_{2}^{*}$ or $B_{1}^{*}<B_{2}^{*}<B_{\sharp}$, which is equivalent to $1<\mathcal{C}_{a 1}<\mathcal{C}_{a 2}$ or $\mathcal{C}_{a 1}<\mathcal{C}_{a 2}<1$;

- $\left(B_{1}^{*}, H_{11}^{*}\right)$ and $\left(B_{2}^{*}, H_{22}^{*}\right)$ are always unstable when they exist;

- $\left(B_{i}^{*}, H_{i j}^{*}\right)$ (with $i, j \in\{1,2\}$ and $\left.i \neq j\right)$ is asymptotically stable when it exists if $\delta^{\prime}\left(B_{i}^{*}\right)+$ $\phi^{\prime}\left(B_{i}^{*}\right) H_{i j}^{*}>0$.

For all concerned equilibria, stability is lost if the corresponding inequalities are strictly reversed.

We summarize the stability situation in Fig. 3 and Table 3 when both $B_{1}^{*}$ and $B_{2}^{*}$ exist; if only $B_{1}^{*}$ does, the situation is identical to that of Fig.2 and Table 2. Fig.3 groups together the linear functions $\delta\left(B_{i}^{*}\right)+\phi\left(B_{i}^{*}\right) H$ generated by $B_{1}^{*}$ and $B_{2}^{*}$, with the one corresponding to 
$B_{1}^{*}$ being lower than the other in $H=0$ because $\delta\left(B_{1}^{*}\right)=\delta\left(\mathcal{C}_{a 1} B_{\sharp}\right)<\delta\left(B_{2}^{*}\right)=\delta\left(\mathcal{C}_{a 2} B_{\sharp}\right)$; the position of these values with respect to $r_{B}(0)=\delta\left(B_{\sharp}\right)$ is then dictated by the values of the $\mathcal{C}_{a i}$ compensation indices. This relationship between the $\mathcal{C}_{a i}$ values and the positions of the curves with respect to each other allows then for the construction of Table 3 which gives all the possible equilibria configurations for different combinations of $\mathcal{C}_{a i}$ and $\mathcal{C}_{s i}$. Note however that, when $\mathcal{C}_{a 2}>1$ and $\mathcal{C}_{s 2}>1$, the equilibria configurations are not directly established. As will be illustrated in the following bifurcation analysis, an additional relationship between $\mathcal{C}_{a i}$ and $\mathcal{C}_{s i}$ helps determine which case occurs; for instance, for given $\mathcal{C}_{s i}>1$, this relationship will help discriminate between cases (ii) and (iii) of Figure 3 by determining for which (threshold) value the long-dashed line and the solid curve are tangent.

In Fig.3, we represent the special case where $\phi(B)$ is constant, which forces $\mathcal{C}_{s 1}=\mathcal{C}_{s 2}$. This guarantees the asymptotic stability of the equilibria $\left(B_{1}^{*}, H_{12}^{*}\right)$ and $\left(B_{2}^{*}, H_{21}^{*}\right)$ when they exist and imposes the linear functions being parallel. For $\phi(B)$ decreasing, the slope of the $B_{1}^{*}$ linear function would be larger than that of the one corresponding to $B_{2}^{*}$, so that both can cross and the existence and ordering of the $H_{i j}^{*}$ equilibria can be changed, while the stability of $\left(B_{1}^{*}, H_{12}^{*}\right)$ and $\left(B_{2}^{*}, H_{21}^{*}\right)$ could be lost. However, non-constant $\phi(B)$ functions can only generate one case that is qualitatively different from what is illustrated on Figure 3 , case (iii-bis) of Table 3 . This case occurs when $\phi\left(B_{1}^{*}\right)$ is much larger than $\phi\left(B_{2}^{*}\right)$ so that the slope of the long-dashed line is much larger than that of the short-dashed line; starting from Fig.3-(vi) and increasing the slope of the long-dashed line, its intersections with $r_{B}(H)$ could be lost without eliminating those of the other line. We term this case (iii-bis) since, like case (iii), it has a stable herbivore-free equilibrium and can have a stable overcompensation equilibrium.

Table 3 and Fig.3 regroup all the possible biological cases arising from the cumulative effect of direct compensation and resistance defences. We note that cases (i), (ii), and (iii) yield identical situations to the ones exposed in the "Direct compensation" case. On the basis of case (i), undercompensation only, the plant defences help to create two new situations: one where the plants either successfully reject the invasion or reach an undercompensation 
equilibrium (case (iv)) and one where the plants either control the herbivore invasion and reach overcompensation or get seriously damaged by a large herbivore population (case (v)). The last new case, case (vi), occurs when the herbivore-free equilibrium is low, and the herbivore invasion triggers a strong positive plant response, with the plants being able to sustain both large and low herbivore densities while overcompensating.

\section{Simulations}

In order to better understand the different equilibria and stability patterns produced by the model, we opted for an explicit analysis for special forms of functions in model (1). We considered the particular model

$$
\left\{\begin{array}{l}
\dot{B}=r\left(1+\frac{H}{\beta}\right)\left(1-\frac{H}{\gamma}\right) B-\delta B^{2}-\phi B H, \\
\dot{H}=\frac{B}{\kappa B^{2}+\nu B+\theta} H-\mu H .
\end{array}\right.
$$

where $\kappa=0$ yields the "Direct compensation" case and $\kappa>0$ includes the resistance phenomenon. All other parameters are positive. Plants response to herbivory is handled through the function $r_{B}(H)=r\left(1+\frac{H}{\beta}\right)\left(1-\frac{H}{\gamma}\right)$, which is a concave, increasing, then decreasing, function of $H$ as long as $r_{B}^{\prime}(0)=\beta^{-1}-\gamma^{-1}>0$. For the following analysis, we thus assumed $\beta<\gamma$. Maintenance is assumed linear $(\delta(B)=\delta B)$, while function $\phi(B)$ is assumed to be constant and equal to $\phi$. The latter implies that positive equilibria cannot lose their stability through a Hopf bifurcation, so that positive equilibria are either saddle points or LAS in this particular example, and that there is a single short-term compensation index $\mathcal{C}_{s}=\mathcal{C}_{s 1}=\mathcal{C}_{s 2}$. All these functions fulfill the general modelling hypotheses exposed in Section 2. These particular forms allow one to compute $\mathcal{C}_{s}$ explicitly as: $\mathcal{C}_{s}=\frac{r}{\beta \phi}\left(1-\frac{\beta}{\gamma}\right)$. Since the condition $r<\beta \phi$ restricts $\mathcal{C}_{s}$ to values smaller than 1 , we concentrated on the more interesting situation where $r>\beta \phi$.

\subsection{Bifurcation diagrams}

\subsubsection{Direct compensation}

The herbivore-free equilibrium is $\left(B_{\sharp}, 0\right)=\left(\frac{r}{\delta}, 0\right)$. With $\kappa=0$ and $\mu<\frac{1}{\nu}$, the $\dot{H}=0$ equation can only yield a single $B^{*}=\alpha^{-1}(\mu)>0$. The corresponding herbivore level $H^{*}$ is 
then the solution of

$$
r\left(1+\frac{H}{\beta}\right)\left(1-\frac{H}{\gamma}\right)-\delta B^{*}-\phi H=0
$$

Rearranging the terms and injecting $\mathcal{C}_{s}$ and $\mathcal{C}_{a}=\frac{\delta B^{*}}{r}$ in this equation we get

$$
H^{2}-(\gamma-\beta)\left(1-\frac{1}{\mathcal{C}_{s}}\right) H+\beta \gamma\left(\mathcal{C}_{a}-1\right)=0
$$

We already know what the number of equilibria is and what they correspond to when $\mathcal{C}_{a}<1$ and/or $\mathcal{C}_{s}<1$ (see Tab. 2). We are then left to discriminate between cases (ii) and (iii) of Table 2 when $\mathcal{C}_{a}>1$ and $\mathcal{C}_{s}>1$. In this situation, equation (9) has two positive roots (case (iii)) if its discriminant is positive, that is if

$$
(\gamma-\beta)^{2}\left(1-\frac{1}{\mathcal{C}_{s}}\right)^{2}-4 \beta \gamma\left(\mathcal{C}_{a}-1\right)>0
$$

or, taking into account the constraint $\mathcal{C}_{a}>1$

$$
1<\mathcal{C}_{a}<1+\frac{(\gamma-\beta)^{2}}{4 \beta \gamma}\left(1-\frac{1}{\mathcal{C}_{s}}\right)^{2}
$$

This inequality, which differentiate between cases (ii) and (iii) of Table 2, only depends on $\beta$ and $\gamma$, which confirms that most information was in $\mathcal{C}_{s}$ and $\mathcal{C}_{a}$. It transforms into

$$
1<\frac{B^{*}}{B_{\sharp}}<1+\frac{1}{4 r^{2} \beta \gamma}(r(\gamma-\beta)-\phi \beta \gamma)^{2}
$$

that we only study for $\mathcal{C}_{s}=\frac{r}{\beta \phi}\left(1-\frac{\beta}{\gamma}\right)$ larger than 1 .

We are interested in comparing different levels of plant responses to herbivory. Increasing parameter $\gamma$ easily creates a family of ordered $r_{B}(H)$ curves featuring increasing plant direct compensation capacity with otherwise comparable properties (such as the herbivore-free growth rate $r_{B}(0)=r$ ). Fixing all other parameters, we then see the right-hand-side of (10) as an increasing function of $\gamma, F(\gamma)$, which is defined only for $\gamma>\frac{r \beta}{r-\phi \beta}$, since here we have $\mathcal{C}_{s}>1$. Taking into account that $B^{*}=\alpha^{-1}(\mu)$, model (8) has two overcompensation equilibria, only one of which is stable, if

$$
\alpha\left(B_{\sharp}\right)<\mu<\alpha\left(B_{\sharp} F(\gamma)\right)
$$


with $\gamma>\frac{r \beta}{r-\phi \beta}$ (case (iii), shaded area in Figure 4); if $\mu>\alpha\left(B_{\sharp} F(\gamma)\right)$ then model (8) has no positive equilibrium (case (ii)); if $\mu<\alpha\left(B_{\sharp}\right)$ then $\mathcal{C}_{a}<1$ which corresponds to case (i) with one stable undercompensation equilibrium. When $\gamma<\frac{r \beta}{r-\phi \beta}$, then $\mathcal{C}_{s}<1$ and the position of $\mu$ with respect to $\alpha\left(B_{\sharp}\right)$ discriminates between cases (i) and (ii) .

Thus, we were able to completely characterize model (8) dynamics through parameters $\gamma$ and $\mu$, which characterize plant reponses to herbivory and herbivore mortality, respectively. These developments are summarized in Figure 4 and further discussed in the Discussion section of the article.

\subsubsection{Direct compensation and Resistance}

In the "Direct compensation and Resistance" model, we will again use $\mu$ and $\gamma$ as bifurcation parameters. Here, equation $\mu=\alpha(B)$ potentially generates two equilibrium solutions $B_{1}^{*}$ and $B_{2}^{*}$, corresponding respectively to the increasing and decreasing parts of $\alpha(B)$. If $\mu>\alpha\left(B_{m}\right)$, no equilibrium exists; we then discuss the situations where $\mu<\alpha\left(B_{m}\right)$. Throughout, different situations will occur depending whether $B_{\sharp}<B_{m}$ or $B_{\sharp}>B_{m}$, which are illustrated in Figure 5 (a) and Figure 5 (b), respectively.

We will first consider the equilibria built on $B_{1}^{*}$. If $\mathcal{C}_{a 1}<1$, there is a stable undercompensation equilibrium. This translates into $B_{1}^{*}<B_{\sharp}$, which is trivially satisfied for all $\mu<\alpha\left(B_{m}\right)$ when $B_{\sharp}>B_{m}$ and otherwise yields $\mu<\alpha\left(B_{\sharp}\right)$.

Otherwise, the essential issue is whether $B_{1}^{*}$ can generate two herbivore equilibrium solutions (an unstable and a stable overcompensation one) which, as previously, is monitored by equation (10) which yields

$$
B_{\sharp}<B_{1}^{*}<B_{\sharp} F(\gamma)
$$

The left inequality has two consequences: it can not be verified when $B_{\sharp}>B_{m}$ (otherwise $B_{m}<B_{1}^{*}$ which is a contradiction), and, if $B_{\sharp}<B_{m}$, it imposes $\mu>\alpha\left(B_{\sharp}\right)$ through the application of $\alpha$ to the inequality. The right inequality then becomes

$$
\gamma>F^{-1}\left(\frac{\alpha_{i n c}^{-1}(\mu)}{B^{\sharp}}\right)
$$

where $\alpha_{i n c}^{-1}($.$) is the inverse of the increasing part of \alpha(B)$. As in the Direct compensation 
case, this situation can thus be illustrated using the threshold curve

$$
\mu=\alpha\left(B_{\sharp} F(\gamma)\right),
$$

initiated at $(\gamma, \mu)=\left(\frac{r \beta}{r-\phi \beta}, \alpha\left(B_{\sharp}\right)\right)$, that is at $\mathcal{C}_{s}=1$. The $B_{1}^{*}$ overcompensation equilibrium then exists in the whole region on the right of the increasing part of the curve when this part exists, that is when $B_{\sharp}<B_{m}$ (shaded area in Figure 5 (a) with $\mu>\alpha\left(B_{\sharp}\right)$ ).

As for $B_{2}^{*}$, it never generates a stable undercompensation equilibrium, as can be seen in Table 3 ; only the case $\mathcal{C}_{a 2}>1$ then matters and the situation with two equilibria (including a stable overcompensation one) is again obtained from (10)

$$
B_{\sharp}<B_{2}^{*}<B_{\sharp} F(\gamma) .
$$

The left inequality only yields a constraint when $B_{\sharp}>B_{m}$ (otherwise it is trivial): applying $\alpha$, which is then decreasing at $B_{\sharp}$ and $B_{2}^{*}$, it then imposes $\mu<\alpha\left(B_{\sharp}\right)$. The right inequality is then satisfied when

$$
\gamma>F^{-1}\left(\frac{\alpha_{d e c}^{-1}(\mu)}{B^{\sharp}}\right)
$$

where $\alpha_{d e c}^{-1}($.$) is the inverse of the decreasing part of \alpha(B)$. This threshold always exist even if $B_{m}<B_{\sharp}$. In that case, the curve arising from this inequality is defined only for $\mu<\alpha\left(B_{\sharp}\right)$, while it is defined for all $\mu<\alpha\left(B_{m}\right)$ otherwise. The $B_{2}^{*}$ overcompensation case then occurs on the right of the decreasing part of the curve (12) (shaded regions right to the decreasing part of the black curve on Figures 5 (a) and (b)).

The link between Figures 5 (a) and (b) and Table 3 can then simply be made by analyzing the number and types of positive equilibria in the various regions. For instance, the regions where no equilibrium has been found through the previous analysis, such as left to the increasing part of the black curve and above $\mu=\alpha\left(B_{m}\right)$ in Figure $5(\mathrm{a})$, correspond to case (ii). Also, in the shaded area right to the decreasing part of the black curve with $\mu$ between $\alpha\left(B_{\sharp}\right)$ and $\alpha\left(B_{m}\right)$, both $B_{1}^{*}$ and $B_{2}^{*}$ generate overcompensation equilibria, which corresponds to case (vi). The remainder of the links is made accordingly and illustrated on Figures 5. 


\subsection{Phase plane diagrams}

To illustrate the dynamical behaviour produced by model (8) in the "Direct compensation" and in the "Direct compensation and Resistance" cases, we computed typical phase plane diagrams in two specific cases producing bi-stable patterns (Figure 6). Bi-stability indicates that the long-term behaviour of the model is determined by a critical relationship between plants and herbivores densities at the onset of herbivory. Phase plane analysis helps to determine the conditions leading to one or the other long term behaviour. Figure 6 was obtained from fairly standard phase plane analysis methods that are not detailed any further.

Figure 6 (a) figures case (iii), in which both the herbivore-free equilibrium and an overcompensation equilibrium are asymptotically stable, as simulated from the "Direct compensation" version of model (8). Other cases relevant to this model, ensuring either the existence and stability of the herbivore-free equilibrium alone (case (ii)) or of an undercompensation one (case (i)), are easily interpreted and thus do not require phase plane analyses. As for case (iii), Figure 6 (a) shows that if the initial herbivore density is large enough, it can trigger a sufficiently strong positive response of the plants, which can sustain a herbivore population in the long term and leads to plant-herbivore co-existence at an overcompensation equilibrium (mutualistic interaction). If, however, the initial herbivore density is too small, the positive response of the plants is not strong enough to make it possible for the herbivores to survive; the latter are then doomed to extinction because of starvation.

We investigated the "Direct compensation and Resistance" model (8) in Figure 6 (b), focusing on case (v). Indeed, we considered that the $B_{\sharp}<B_{m}$ situation, although it yielded interesting theoretical situations such as tri-stability (case (vi)), was biologically unlikely. $B_{m}$ is the plant biomass level above which plant defences start acting negatively against herbivores. It is most plausible that fully grown plants at the herbivore-free equilibrium $\left(B=B_{\sharp}\right)$ have efficient defences against herbivores, at least if they can express defences at all (otherwise we are back to the "Direct compensation" model). Concentrating on $B_{\sharp}>B_{m}$, we were thus left with cases (i), (ii), (iv) and (v). The two former do not pose any further difficulties than in the "Direct compensation" case, case (iv) is similar to case (iii) illustrated 
in Figure 6, except that the co-existence equilibrium is an undercompensation one.

Case (v) is characterized by one overcompensation and one undercompensation equilibrium, both of which being asymptotically stable; small initial herbivore densities lead to overcompensation and large ones to undercompensation (Figure 6 (b)). This can be explained thus: when the initial herbivore density is small, the direct herbivory effect is small too. As both plant biomass and herbivore density increase, the former reaches a level beyond which herbivore growth is limited by plants resistance abilities before the direct herbivory effect takes its toll. Hence, even if the positive response of plants remains small, the balance between herbivory and plants response is in favour of the latter, plants and herbivores co-exist at an overcompensation equilibrium (mutualistic interaction). When the initial herbivore density is large, the direct herbivory effect is strong so that plants cannot make up for it and grow well; the balance between herbivory and plants response is in favour of the former. Herbivores are then rarely confronted with negative effects of plants resistance that are only tangible at high plant biomass levels. Plants and herbivores then reach an undercompensation equilibrium, i.e. the usual situation where plants suffer from herbivore presence while herbivores benefit from plants (antagonistic interaction).

Case (v) corresponds thus to a situation where, depending on the respective initial densities of consumers and resources, the outcome of the consumer-resource interaction may be antagonistic or mutualistic. This property is for instance qualitatively similar to the results reported by (Poveda et al., 2010) on the interaction between potato plants and the Guatemalan potato moth, where large and small populations of the pest lead to small and large potato plants, respectively.

\section{Discussion}

This contribution focused on plant-herbivore interactions with a particular attention given to understanding the conditions under which plants can benefit from the presence of herbivores. Plants response to herbivory can be broadly categorized into tolerance and resistance strategies. Given that plant resistance essentially impacts herbivore development, it can barely benefit to plants as compared with a herbivore-free situation. We thus first 
concentrated on tolerance in the form of direct plant compensation, by studying a simple plant-herbivore model in which plants growth rate may respond positively to the presence of herbivores (compensation effect or $\mathrm{HOH}$, Figure 1). We then considered that plants may also resist herbivory, assuming that herbivores growth rate was a non-monotonous function of plants density. This progressive integration of different biological mechanisms into a basic plant-herbivore model allows for a better understanding of the effects of herbivory, plant tolerance, and plant resistance, and their interactions.

As illustrated in the tables and bifurcation diagrams, many different patterns of dynamics can arise, depending on the model hypotheses (Direct compensation versus Direct compensation and Resistance) as well as on parameter values. We will essentially comment on long-term dynamics, hence apparent compensation. Three cases occur in both models: only plants can survive (case (ii), herbivores die out due to starvation and the possible important expression of plant defences); plants and herbivores co-exist in an undercompensation situation (case (i), plants cannot make up for herbivory); plants and herbivores either coexist in an overcompensation situation, or only plants can survive (case (iii)). Three cases are specific to the Direct compensation and Resistance model: plants and herbivore either co-exist in an undercompensation situation, or only plants can survive (case (iv)); plants and herbivore co-exist either in an under- or over-compensation situation (case (v)); plants and herbivore co-exist either in one among two overcompensation situations, or only plants can survive (case (vi)). Herbivore-free and undercompensation situations can be seen as the classical situations where herbivores have neutral or negative effects on plant biomass. Yet, the remarkable situations corresponding to overcompensation by plants are also evidenced in various cases (iii, v, vi), among which the more plausible scenario where $B_{\sharp}>B_{m}$ (case (v), see section 4.2).

We can evaluate the impact of plant compensation by comparing the Rosenzweig-McArthur (RMA) model (Rosenzweig and MacArthur, 1963) to the direct compensation model. The RMA model is well known for generating stable periodic trajectories through the paradox of enrichment; the same property holds in both our models since stable positive equilibria may 
be destabilized through a Hopf bifurcation when $\delta^{\prime}\left(B^{*}\right)+\phi^{\prime}\left(B^{*}\right) H^{*}<0$. In the following, we will concentrate the discussion on the case where positive equilibria are not destabilized by that mechanism. The comparison between the RMA and direct compensation models can be achieved by noting that the RMA model corresponds to $r_{B}(H)=r_{B}(0)$ for all $H$. A quick analysis of Proposition 1 and Figure 2 for the RMA model shows that the only possible situations are survival of plants only (case (ii)) and co-existence equilibria with reduced plant biomass as compared with the herbivore-free situation (case (i)). When the direct compensation mechanism is added to the RMA model, case (ii) may either be unchanged, or additionally generate a co-existence situation characterized by plants overcompensation (case (iii)); as for case (i), it remains structurally similar, but Figure 2 (i) shows that herbivore density is well increased $\left(H_{2}^{*}>H_{0}^{*}\right.$, with $H_{0}^{*}$ corresponding to the RMA case). Also, in that case, the level of biomass is not influenced since it is ruled by the $\dot{H}=0$ equation. Thus, the direct compensation mechanism can have three typical apparent compensation consequences: be neutral, if a herbivore free equilibrium stays that way, be positive for both plants and herbivores if overcompensation occurs or, be only profitable to the herbivore in the case with undercompensation. A similar comparison between the Direct compensation and Resistance model and a Resistance-only model, such as the one developed by Freedman and Wolkowicz (1986), shows that the same properties hold most of the time. The only exception stems from case (i) in the Resistance-only model, which may generate overcompensation in case (v) in the Direct compensation and Resistance model. In that situation, plants and herbivore may co-exist at high plant biomass levels, which holds herbivores to low density thanks to a compensation-mediated expression of resistance.

An important novelty of our model is that, under the direct compensation hypothesis, it predicts that apparent plant overcompensation can occur. This result is not frequent in plant-herbivore models, which, if they assume or predict positive responses of plants, usually conclude that, at equilibrium, plants reach lower densities confronted to herbivores than in a herbivore-free situation, i.e. undercompensation (Loreau, 1995; de Mazancourt et al., 1998; Ryrie and Prentice, 2011; Fukui et al., 2012). Without an explicit modelling of the herbi- 
vore population, Dyer et al. (1986) however found overcompensation situations stemming from constant herbivory. de Mazancourt (2001) also unveiled a similar property in an ecoevolutionary study on plant-herbivore dynamics. Plant overcompensation under herbivory actually means that plants and herbivores have a mutualistic relationship: plants benefit from herbivore presence and herbivore would not survive in the absence of plants. Whether such a mutualism can occur within a basically antagonistic interaction has been the matter of heated debates over the years (Belsky, 1986; Paige and Whitham, 1986; Belsky et al., 1993; Agrawal, 2000), although there was much evidence supporting it (see the Introduction). In our model, plant-herbivore mutualism can never be the unique stable situation: it is always coupled with a stable neutral or antagonistic outcome of the interaction. Thus, depending on the initial plant and herbivore levels, the plant-herbivore model may present mutualistic patterns or not (Figure 6).

In a more general ecological framework, mutualism has recently been shown as a possible outcome of basically antagonistic resource-consumer interactions (Holland and DeAngelis, 2009, 2010). As in the present study, such mutualism relies on the positive response of resources to the presence of consumers, and it occurs as well as particular stable instances of multi-stable dynamics. In the resource-consumer theory proposed by Holland and DeAngelis (2009, 2010), the possible outcomes are generally characterized by a co-existence situation (which may be mutualistic) and the extinction of one or the other species. In their work, extinction occurs because of overexploitation of one species by the other, and not like here, because of starvation of the consumers. Overexploitation of plants by herbivores would also be possible in our framework, for instance if other food sources were available to the herbivores; at the same time, this would prevent them from extinction. Another original feature of our model is that it can also generate multiple stable equilibria at which both species co-exist. For instance, depending on the initial conditions, the plant-herbivore model can display stable antagonistic and mutualistic co-existence equilibria. Such a pattern is, as far as we know, a new theoretical finding. This does not mean that it is biologically unlikely: the potato-potato moth experiment reported by Poveda et al. (2010) appears as a good real 
life example of such dynamics.

That herbivores may benefit plants and that both may enjoy mutualistic relationships could have important applications in agronomy and crop pest management. The idea, originally proposed by Harris (1974), is that (some) agricultural crops may require a certain level of pests to achieve maximum yield. For instance, Dyer et al. (1993) reported that cotton crops in China had a better yield under moderate infestation by the cotton boll worm than without the pest. At that time, ensuring a certain level of worms within cotton crops even became a recommended practice, which resulted in an increase in cotton production (Dyer et al., 1993). Apart from cotton plants, many other agronomic plants have the capacity to respond positively to herbivory (Trumble et al., 1993; Thomson et al., 2003; Leimu and Koricheva, 2006). A potential application of our modeling approach is that it could give indices at how pest management could be improved by taking into account or even exploiting the overcompensation potential of some plants, to gain productivity. Our bifurcation and phase plane analyses show that two buttons could and should be triggered. On the one hand, the modification of model parameters to make overcompensation possible (case (iii) in Tab. 2, and cases (iii)-(v)-(vi) in Tab. 3). On the other hand, even when overcompensation conditions are fulfilled, the density dependence governing its realization might require that action be taken to bring the plant biomass/pest density into a region where overcompensation will actually take place (e.g. below the separatrix in Fig. $6(\mathrm{~b}))$; this could for instance be achieved by some direct action on the pest population.

Since most of the outcome of the plant-herbivore dynamics depends on the $\mathcal{C}_{a}$ and $\mathcal{C}_{s}$ threshold parameters, how to modify original control parameters in the model mainly relies on them; these controls are related either to the crop parameters, the pest parameters, and/or to the plant-pest interaction parameters. In Section 4.1, we have extracted, and will only discuss, two of those: $\mu$, the pest death rate, and $\gamma$, which defines the intensity of the plant response to herbivory. Traditionally, pest control management recommends an increase of the pest death-rate to reduce biomass damage and, if massive enough, this strategy could lead to pest eradication (into region (ii) of Figures 4 and 5); however, if the 
initial parameters are in region (i), a less pronounced increase of $\mu$ could lead the plantpest system into the overcompensation regions of the Figures (shaded areas), provided $\gamma$ is large enough. In addition to this "traditional" pest control, our results highlight other control strategies, some of them seeming paradoxical: indeed, reaching overcompensation in Figures 4 and 5 from regions (ii) and (iv), where the plants by themselves are able to fight off the pest invasion, can require to decrease the pest death-rate. Additionally, or instead, the $\gamma$ parameter can be played with; in many instances, if overcompensation is originally not possible, an increase of this plant characteristic parameter can lead the system into the shaded area. This can be achieved by an appropriate management of Direct compensation traits in the crop strain choice. In summary, if a plant-pest mutualistic interaction is possible, increased productivity can be obtained by not hitting the pest too hard or, if necessary, favoring it; also, when choosing or modifying a plant strain, it is best to pick the one with the largest compensation capacity. These are not surprising given that the aim is to exploit mutualism; our model confirms that, if the conditions are right, such actions indeed ensure a productivity increase.

Among plant defences, plant resistance to herbivory has been known for centuries, long before its conceptualization, and has been taken advantage of for crop protection ever since, leading to an arms race between pests and farmers. On its part, plant compensation has been thought to be a powerful tool in crop-pest management for about 40 years. It is a potentially long-term crop protection solution due to the lack of evidence of compensation breakdown through herbivore evolution (Fornoni, 2011) as well as the limited impact it has on nontarget species. However, the advances in the knowledge of the underlying mechanisms and the development of its use have been slow. The present study confirmed that compensation could be exploited in various ways in agronomy and that the current research effort on these mechanisms should be intensified.

\section{Acknowlegments}

AMAP (Botany and Computational Plant Architecture) is a joint research unit which associates CIRAD (UMR51), CNRS (UMR5120), INRA (UMR931), IRD (2M123), and Mont- 
pellier 2 University (UM27); http://amap. cirad.fr.

AL benefited from a $\mathrm{PhD}$ schorlarship funded by CIRAD. This research was supported in part by the European Commission through the $7^{\text {th }}$ Framework Program (PURE project, contract number 265865).

Agrawal, A. A., 2000. Overcompensation of plants in response to herbivory and the byproduct benefits of mutualism. Trends in plant science 5 (7), 309-313.

Andrews, J., 1968. A mathematical model for the continuous culture of microorganisms utilizing inhibiting substrates. Biotech. Bioeng. 10, 707-723.

Arimura, G., Kost, C., Boland, W., 2005. Herbivore-induced, indirect plant defences. Biochimica et biophysica acta 1734 (2), 91-111.

Belsky, A., 1986. Does herbivory benefit plants ? a review of the evidence. The American Naturalist 127 (6), 870-892.

Belsky, A., Carson, W., Jensen, C., Fox, G., 1993. Overcompensation by plants : herbivore optimization or red herring? Evolutionary Ecology 7, 109-121.

Brown, B., Allen, T., 1989. The importance of scale in evaluating herbivory impacts. Oikos $54(2), 189-194$.

Center, T., Van, T., Dray Jr, F., Franks, S., Rebelo, M., Pratt, P., Rayamajhi, M., 2005. Herbivory alters competitive interactions between two invasive aquatic plants. Biological control 33, 173-185.

Crawley, M., 1989. Insect herbivores and plant population dynamics. Annual Review of Entomology 34, 531-564.

de Mazancourt, C., 2001. Can the evolution of plant defense lead to plant-herbivore mutualism? American Naturalist 158, 109-123.

de Mazancourt, C., Loreau, M., Abbadie, L., 1998. Grazing optimization and nutrient cycling : when do herbivores enhance plant production ? Ecology 79 (7), 2242-2252. 
DeAngelis, D., 1992. Dynamics of nutrient cycling and food webs. Chapman \& Hall.

Dyer, M., 1975. The effects of red-winged blackbirds (Agelaius phoenicus 1.) on biomass production of corn grain (Zea mays 1.). Journal of Apllied Ecology 12, 719-726.

Dyer, M., DeAngelis, D., Post, W., 1986. A model of herbivore feedback on plant productivity. Mathematical biosciences 79, 171-184.

Dyer, M., Turner, C., Seastedt, T., 1993. Herbivory and its consequences. Ecological applications $1,10-16$.

Dyer, M. I., Moon, A. M., Brown, M. R., Crossley, D. A., 1995. Grasshopper crop and midgut extract effects on plants: an example of reward feedback. Proceedings of the National Academy of Sciences 92 (12), 5475-5478.

Feng, Z., Liu, R., DeAngelis, D., 2008. Plant-herbivores interactions mediated by plant toxicity. Theoretical Population Biology 73, 449-459.

Feng, Z., Qiu, Z., Liu, R., DeAngelis, D., 2011. Dynamics of a plant-herbivore-predator system with plant toxicity. Mathematical Biosciences 229, 190-204.

Fernandes, G., 1994. Plant mechanical defenses against insect herbivory. Revta bras. entomology 38 (2), 321-433.

Fornoni, J., 2011. Ecological and evolutionary implications of plant tolerance to herbivory. Functional Ecology 25 (2), 399-407.

Freedman, H., Wolkowicz, G., 1986. Predator-prey systems with group defense: The paradox of enrichment revisited. Bulletin of Mathematical Biology 48, 493-508.

Fukui, S., Miki, T., Shimada, M., 2012. Consumers can enhance ecosystem productivity and stability in changing environements. Population Ecology 54, 177-186.

Gibson, R., Pickett, J., 1983. Wild potato repels aphids by release of aphid alarm pheromone. Nature 302, 608-609. 
Haldane, J., 1930. Enzymes. Longmans, London.

Hanley, M., Lamont, B., M.M, F., C.M., R., 2000. Plant structural traits and their role in anti-herbivore defence. Perspectives in Plant Ecology, Evolution and Systemics 8, 157-178.

Harris, P., 1974. A possible explanation of plant yield increases following insect damage. Agro-Ecosystems 1, 219-225.

Heil, M., 2010. Plastic defence expression in plants. Evolutionary Ecology 24, 555-569.

Hik, D., Jefferies, R., 1990. Increase the net aboveground primary production of a saltmarsh forage grass : a test of the prediction of the herbivore optimization model. Journal of Ecology 78, 180-195.

Hilbert, D., Swift, D., Detling, J., Dyer, M., 1981. Relative growth rate and the grazing optimization hypothesis. Oecologia 51, 14-18.

Holland, J., DeAngelis, D., 2009. Consumer-resource theory predicts dynamic transitions between outcomes of interspecific interactions. Ecology Letters 12, 1357-1366.

Holland, J., DeAngelis, D., 2010. A consumer-resource approach to the density-dependant population dynamics of mutualism. Ecology 91 (5), 1286-1295.

Holling, C. S., 1959. Some characteristics of simple types of predation and parasitism. The Canadian Entomologist 91, 385-398.

Lebon, A., Mailleret, L., Grognard, F., Dumont, Y., 2012. Modeling plant compensatory effects in plant-insects dynamics. In: Proceedings of the Fourth International Symposium on Plant Growth Modeling, Simulation, Visualization and Applications (PMA 12). pp. $212-219$.

Leimu, R., Koricheva, J., 2006. A meta-analysis of tradeoffs between plant tolerance and resistance to herbivores: combining the evidence from ecological and agricultural studies. Oikos 112 (1), 1-9. 
Lennartsson, T., Nilsson, P., Tuomi, J., 1998. Induction of overcompensation in the field gentian, Gentianella campestris. Ecology 79, 1061-1072.

Li, Y., Feng, Z., Swihart, R., Bryant, J., Huntly, N., 2006. Modeling the impact of plant toxicity on plant-herbivore dynamics. Journal of Dynamics and Differential equations 18 (4), $1021-1042$.

Loreau, M., 1995. Consumers as maximizers of matter and energy flow in ecosystems. The American Naturalist 145 (1), 22-42.

Mabry, C., Wayne, P., 1997. Defoliation of the annual herb Abutilon theophrasti : mechanisms underlying reproductive compensation. Oecologia 111 (2), 225-232.

Marcelis, L., Heuvelink, E., Goudriaan, J., 1998. Modelling biomass production and yield of horticultural crops: a review. Scientia Horticulturae 74 (1-2), $83-111$.

McNaughton, S., 1979. Grazing as an optimization process: grass-ungulate relationships in the serengeti. The American Naturalist 113, 691-703.

McNaughton, S., 1983. Compensatory plant growth as a response to herbivory. Oikos 40, 329-336.

Meyer, G., 1998. Pattern of defoliation and its effect on photosynthesis and growth of goldenrod. Functionnal Ecology 12, 270-279.

Noy-Meir, I., 1975. Stability of grazing systems, application orf predator-prey graphs. Journal of Ecology 63, 459-481.

Nunez-Farfan, J., Fornoni, J., Valverde, P., 2007. The evolution of resistance and tolerance to herbivores. annual review of ecology evolution and systematics $38,541-566$.

Paige, K., Whitham, T., 1986. Overcompensation in response to mammalian herbivory : the advantage of being eaten. The American Naturalist 129 (3), 407-416. 
Paul, M., Foyer, C., 2001. Sink regulation of photosynthesis. Journal of Experimental botany 52 (360), 1383-1400.

Poveda, K., Gomès Jimènez, M., Kessler, A., 2010. The enemy as ally: herbivore-induced increase in crop yield. Ecological Applications 20 (7), 1787-1793.

Quesada, M., Bollman, K., Stephenson, A., 1995. Leaf damage decreases pollen production and hinders performance in Cucurbita texana. Ecology 76 (1), 437-443.

Reich, P., Tjoelker, M., Machado, J.-L., Oleksyn, J., 2006. Universal scaling of respiratory metabolism, size and nitrogen in plants. Nature.

Rosenzweig, M., MacArthur, R., 1963. Graphical representation and stability conditions of predator-prey interactions. The American Naturalist 97, 209-223.

Ryrie, S., Prentice, I., 2011. Herbivores enable plant survival under nutrient limited conditions in a model of grazing system. Ecological modelling 222, 381-397.

Sadras, V., 1996. Coton compensatory growth after loss of reproductive organs as affected by avaibility of resources and duration of recovery period. Oecologia 106, 432-439.

Strauss, S., Agrawal, A., 1999. The ecology and evolution of plant tolerance to herbivory. Trends in ecology and evolution 14 (5), 179-185.

Strauss, S., Rudgers, J., Lau, J., Irwin, R., 2002. Direct and ecological costs of resistance to herbivory. Trends in Ecology and Evolution 17 (6).

Thomson, V., Cunningham, S., Ball, M., Nicotra, A., 2003. Compensation for herbivory by Cucumis sativus through increased photosynthetic capacity and efficiency. Oecologia 134, $167-175$.

Trumble, J., Kolodny-Hirsh, D., Ting, I., 1993. Plant compensation for arthropod herbivory. Annual Reviews Entomology 38, 93-119. 
Williamson, S., Detling, J., Dodd, J., Dyer, M., 1989. Experimental evaluation of the grazing optimization hypothesis. Journal of Range Management 42 (2), 149-152.

Zangerl, A., Hamilton, J., Miller, T., Crofts, A., Oxborough, K., Berenbaum, M., de Lucia, E., 2002. Impact of folivory on photosynthesis is greater than the sum of its holes. PNAS 99 (2), 1088-1091. 


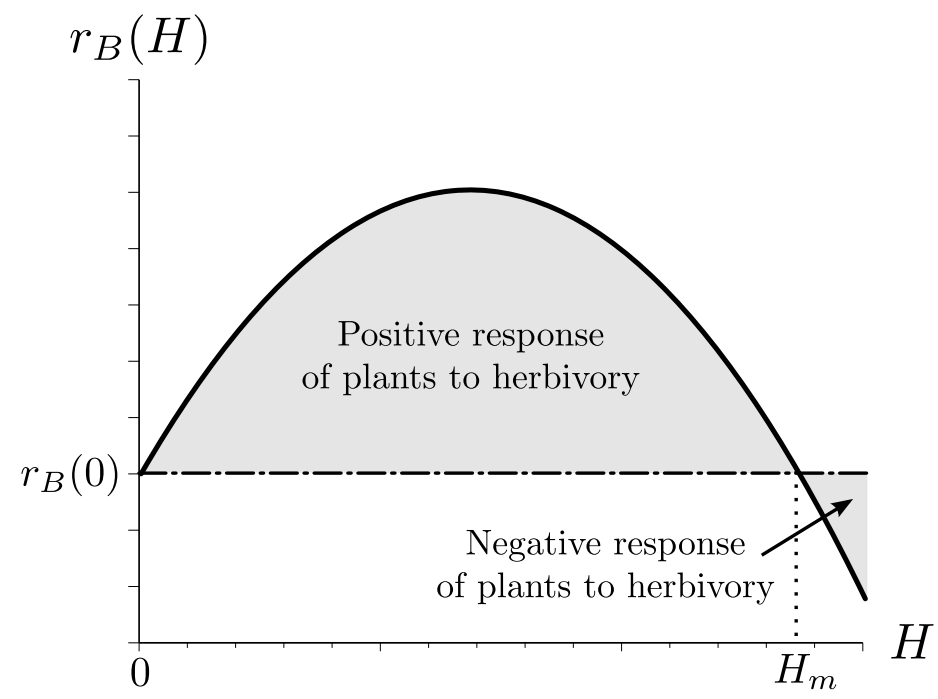

Figure 1: Plant biomass growth rate $r_{B}(H)$ characteristic of the herbivory optimisation hypothesis: for low to moderate levels of herbivory $\left(H<H_{m}\right)$, plant productivity is increased as compared to the herbivory-free case $(H=0)$ and eventually reaches a maximum, but is decreased for large levels of herbivory $\left(H>H_{m}\right)$.

\section{AppendixA. Stability analysis of the equilibria of (1): Direct compensation}

The Jacobian matrix of $(1)$ at a given $(B, H)$ is:

$$
\mathcal{J}(X)=\left(\begin{array}{cc}
r_{B}(H)-\delta^{\prime}(B) B-\delta(B)-\phi^{\prime}(B) B H-\phi(B) H & r_{B}^{\prime}(H)-\phi(B) B \\
\alpha^{\prime}(B) H & \alpha(B)-\mu
\end{array}\right) .
$$

- For the trivial equilibrium $X^{*}=(0,0)$,

$$
\mathcal{J}\left(X^{*}\right)=\left(\begin{array}{cc}
r_{B}(0)-\delta(0) & 0 \\
0 & -\mu
\end{array}\right)
$$

which implies that the equilibrium is a saddle-point, hence unstable, since $r_{B}(0)>\delta(0)$.

- When $X^{*}=\left(B_{\sharp}, 0\right)$ with $B_{\sharp}=\delta^{-1}\left(r_{B}(0)\right)>0$,

$$
\mathcal{J}\left(X^{*}\right)=\left(\begin{array}{cc}
-\delta^{\prime}\left(B_{\sharp}\right) B_{\sharp} & r_{B}^{\prime}(0) B_{\sharp}-\phi\left(B_{\sharp}\right) B_{\sharp} \\
0 & \alpha\left(B_{\sharp}\right)-\mu
\end{array}\right)
$$

which implies that the equilibrium is locally asymptotically stable (LAS) if $\alpha\left(B_{\sharp}\right)<\mu$ or, since $\alpha$ is an increasing function, $B_{\sharp}<B^{*}$, which is equivalent to $\mathcal{C}_{a}>1$. When undercompensation is possible, the herbivore-free equilibrium is unstable. 

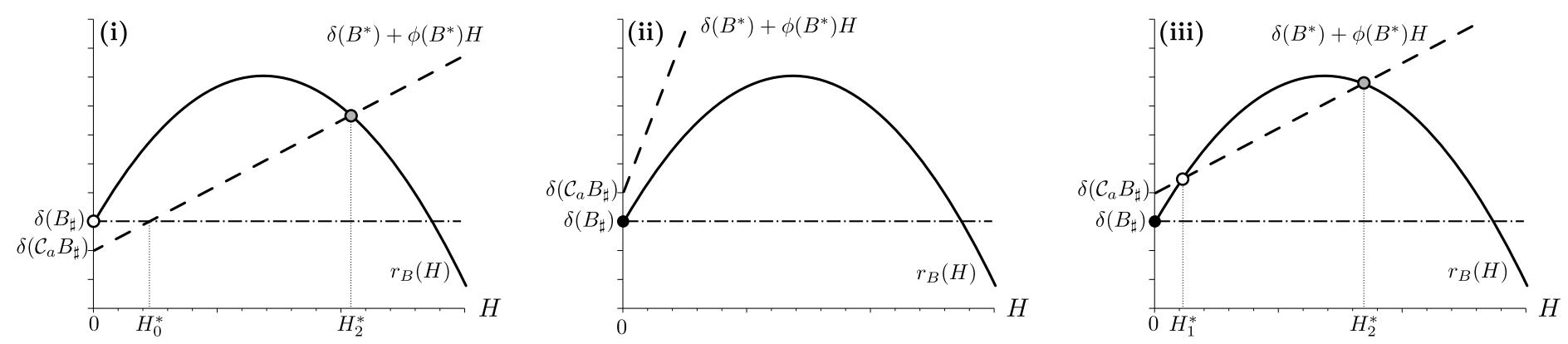

Figure 2: Equilibria and their stability. Different configurations for both sides of equation $(3): r_{B}(H)$ (solid) and $\delta\left(B^{*}\right)+\phi\left(B^{*}\right) H$ (dashed). Case (i): one positive equilibrium $\left(B^{*}, H_{2}^{*}\right)$ with $B^{*}=\mathcal{C}_{a} B_{\sharp}<B_{\sharp}$; case (ii): no positive equilibrium; case (iii): two positive equilibria $\left(B^{*}, H_{1}^{*}\right)$ and $\left(B^{*}, H_{2}^{*}\right)$ with $B^{*}=\mathcal{C}_{a} B_{\sharp}>B_{\sharp}$. White circles correspond to unstable equilibria, black circle to stable equilibria and grey ones to equilibria whose stability depends on an additional condition. The dash-dotted line corresponds to the biomass growthrate without compensation, which only yields an equilibrium $\left(B^{*}, H_{0}^{*}\right)$ in case (i).

- When $X^{*}=\left(B^{*}, H^{*}\right)$, we have $r_{B}\left(H^{*}\right)-\delta\left(B^{*}\right)-\phi\left(B^{*}\right) H^{*}=0$ so that

$$
\mathcal{J}\left(X^{*}\right)=\left(\begin{array}{cc}
-\left(\delta^{\prime}\left(B^{*}\right)+\phi^{\prime}\left(B^{*}\right) H^{*}\right) B^{*} & \left(r_{B}^{\prime}\left(H^{*}\right)-\phi\left(B^{*}\right)\right) B^{*} \\
\alpha^{\prime}\left(B^{*}\right) H^{*} & 0
\end{array}\right) .
$$

and $\left(B^{*}, H^{*}\right)$ is LAS if

$$
\begin{aligned}
& \operatorname{det}(\mathcal{J})=-\alpha^{\prime}\left(B^{*}\right) H^{*} B^{*}\left(r_{B}^{\prime}\left(H^{*}\right)-\phi\left(B^{*}\right)\right)>0 \\
& \operatorname{trace}(\mathcal{J})=-\left(\delta^{\prime}\left(B^{*}\right)+\phi^{\prime}\left(B^{*}\right) H^{*}\right) B^{*}<0
\end{aligned}
$$

The first condition can easily be linked to the slopes of the functions on Figure $2 ; r_{B}^{\prime}\left(H^{*}\right)-\phi\left(B^{*}\right)$ is indeed the difference between the slope of $r_{B}$ and that of $\delta\left(B^{*}\right)+\phi\left(B^{*}\right) H^{*}$ the dashed line. The condition on the determinant is not satisfied at $H_{1}^{*}$ because $r_{B}^{\prime}\left(H_{1}^{*}\right)-\phi\left(B^{*}\right)>0$ there and $\left(B^{*}, H_{1}^{*}\right)$ is then a saddle point. Hence $\left(B^{*}, H_{2}^{*}\right)$ satisfies the determinant condition and is the only possibly asymptotically stable positive equilibrium.

We are then left to check the trace condition for $\left(B^{*}, H_{2}^{*}\right)$. Unless plant consumption by herbivores follows a mass-action principle and $\phi(B)$ is constant, it is not possible to guarantee asymptotic stability for this equilibrium. As soon as $\phi(B)$ is a decreasing function, as would be the case for a Holling II $\phi(B) B$ consumption rate, the trace could be positive. If the slope of $\delta$ is small or that of $\phi$ is large at the equilibrium, 

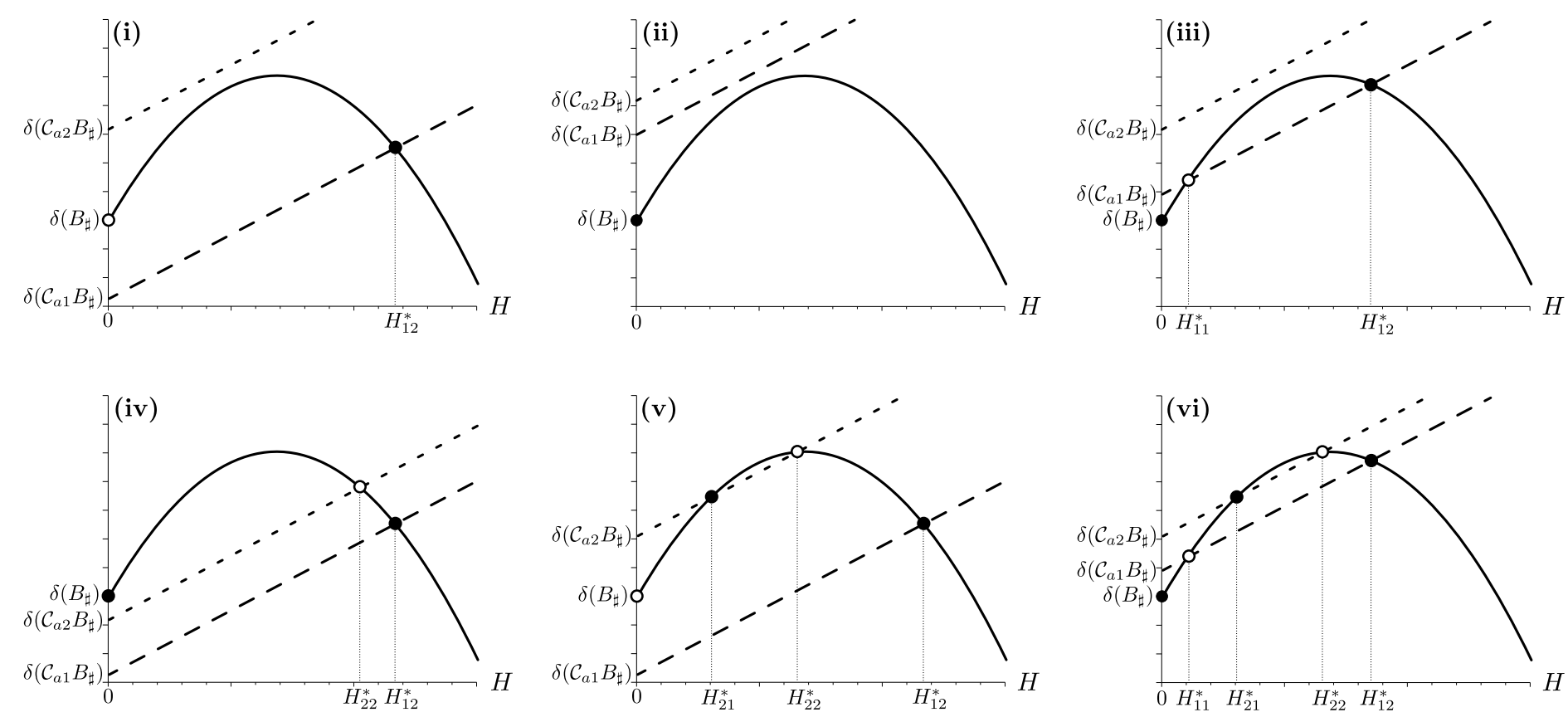

Figure 3: Different configurations for the equilibria. When both $B_{1}^{*}$ and $B_{2}^{*}$ exist, the long and short dashed lines represent $\delta\left(B_{1}^{*}\right)+\phi\left(B_{1}^{*}\right) H^{*}$ and $\delta\left(B_{2}^{*}\right)+\phi\left(B_{2}^{*}\right) H^{*}$, respectively and the solid curve is $r_{B}(H)$. The black circles represent the stable equilibria, and the white ones the unstable ones. Note that $\phi(B)$ was taken constant for this figure so that the linear functions are parallel and the stability of the positive equilibria $\left(B_{1}^{*}, H_{12}^{*}\right)$ and $\left(B_{2}^{*}, H_{21}^{*}\right)$ is guaranteed.

asymptotic stability is lost through a Hopf bifurcation which, as in the Rosenzweig Mac Arthur model (Rosenzweig and MacArthur, 1963), shall generate a stable limit cycle surrounding the equilibrium (results not shown).

\section{AppendixB. Stability analysis of the equilibria of (1): Direct compensation and Resistance}

The stability analysis relies on the same Jacobian matrix $\mathcal{J}(X)$, so that the instability of $(0,0)$ stays unchanged and that of $\left(B_{\sharp}, 0\right)$ still depends on $\alpha\left(B_{\sharp}\right)<\mu$. However, this last condition does not translate as directly as before. Since it is clear that $\alpha(B)>\mu=\alpha\left(B_{i}^{*}\right)$ if and only if $B_{1}^{*}<B<B_{2}^{*}$, it is then direct that $\left(B_{\sharp}, 0\right)$ is LAS if $B_{\sharp}<B_{1}^{*}<B_{2}^{*}$ or $B_{1}^{*}<B_{2}^{*}<B_{\sharp}$, so that $1<\mathcal{C}_{a 1}<\mathcal{C}_{a 2}$ or $\mathcal{C}_{a 1}<\mathcal{C}_{a 2}<1$, both the $B_{i}^{*}$ corresponding to undercompensation or both to overcompensation.

The stability analysis of $\left(B_{1}^{*}, H_{11}^{*}\right)$ and $\left(B_{1}^{*}, H_{12}^{*}\right)$ is identical to that of $\left(B^{*}, H_{1}^{*}\right),\left(B^{*}, H_{2}^{*}\right)$ 


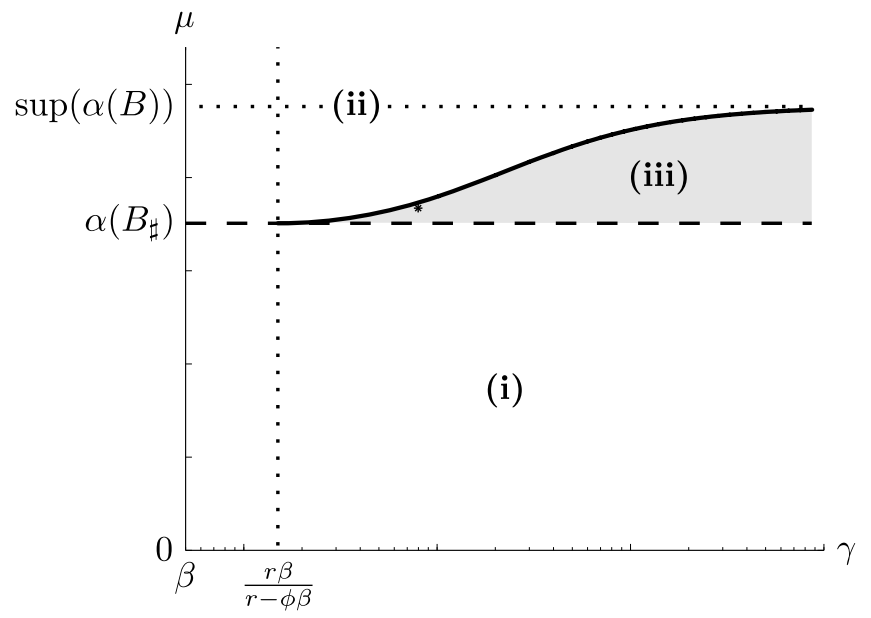

Figure 4: Bifurcation diagrams summarizing the different behaviours of model (8) in the Direct compensation case (see Table 2) as a function of $\gamma$ and $\mu$ (the $\gamma$ axis is in log-scale, while the $\mu$ scale is linear). Parameters are $r=1, \beta=0.5, \delta=3, \phi=\frac{4}{3}$ and $\alpha(B)=\frac{B}{4.2 B+0.5}$. The plain black curve is defined by $\mu=\alpha\left(B_{\sharp} F(\gamma)\right)$. The shaded area corresponds to the existence of overcompensation equilibria. Regions numbering is made in reference to Table 2. The asterisk in panel identifies parameters $\gamma$ and $\mu$ used to compute Figure 6 , case (iii).

since $\alpha^{\prime}\left(B_{1}^{*}\right)>0$ : $\left(B_{1}^{*}, H_{11}^{*}\right)$ is a saddle point and $\left(B_{1}^{*}, H_{12}^{*}\right)$ can be stable. Things are different for $\left(B_{2}^{*}, H_{21}^{*}\right)$ and $\left(B_{2}^{*}, H_{22}^{*}\right)$ : since $\alpha^{\prime}\left(B_{2}^{*}\right)<0$, the slopes conditions coming from the determinant condition in (A.1) are reversed so that it is $\left(B_{2}^{*}, H_{22}^{*}\right)$ that is a saddle point and $\left(B_{2}^{*}, H_{21}^{*}\right)$ is asymptotically stable if $\delta^{\prime}\left(B_{2}^{*}\right)+\phi^{\prime}\left(B_{2}^{*}\right) H_{21}^{*}>0$. 

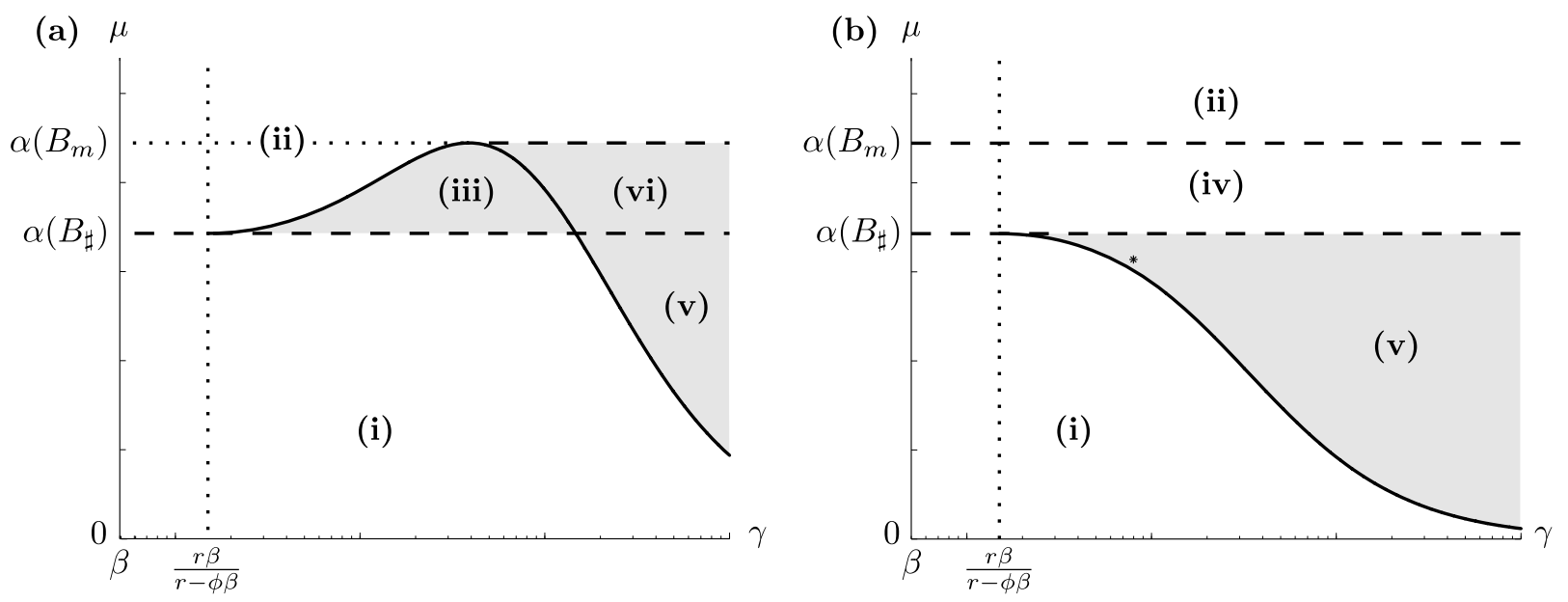

Figure 5: Bifurcation diagrams summarizing the different behaviours model (8) in the "Direct compensation and Resistance" case (see Table 2) in function of $\gamma$ and $\mu$ (the $\gamma$ axis is in log-scale, while the $\mu$ scale is linear). Parameters are $r=1, \beta=0.5, \phi=\frac{4}{3}$ and $\alpha(B)=\frac{B}{B^{2}+2.5 B+0.5}$; such a choice for $\alpha(B)$ makes it essentially comparable to the $\alpha\left(\right.$.) function used in Figure 4, as both functions have similar values for $B<B_{m}=1$ as well as similar suprema. In panel (a), we used $\delta=3$, which illustrates the situation where $B_{\sharp}=\frac{1}{3}<B_{m}=1$, which makes it analogous to Figure 4 up to $\gamma=F^{-1}\left(\frac{B_{m}}{B_{\sharp}}\right)$; in panel (b), we used $\delta=\frac{1}{3}$, which illustrates the situation where $B_{\sharp}=3>B_{m}$. In both panels, the plain black curve is defined by $\mu=\alpha\left(B_{\sharp} F(\gamma)\right)$. The shaded area corresponds to the existence of overcompensation equilibria. Regions numbering is made in reference to Table 3 and explained further in the text. The asterisk in panel (b) identifies parameters $\gamma$ and $\mu$ used to compute Figure 6, case (v). 
(a) $H$

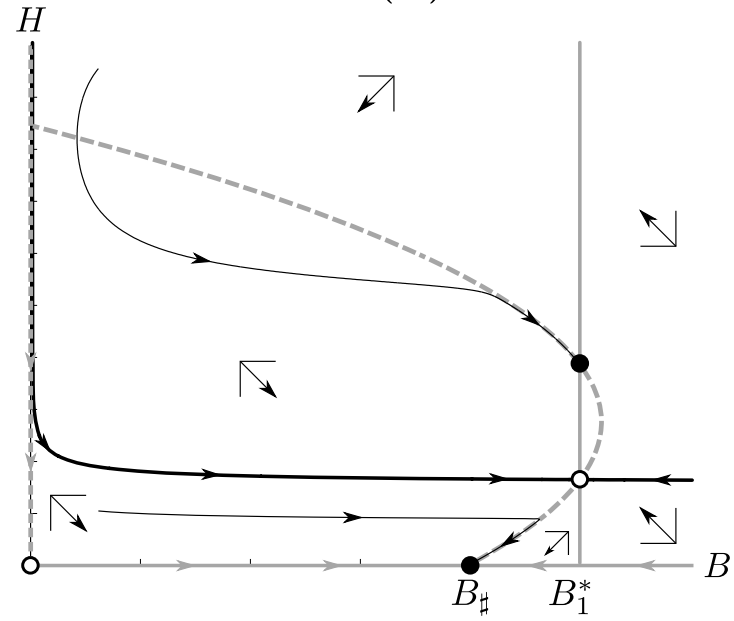

Case (iii)

(b) $H$

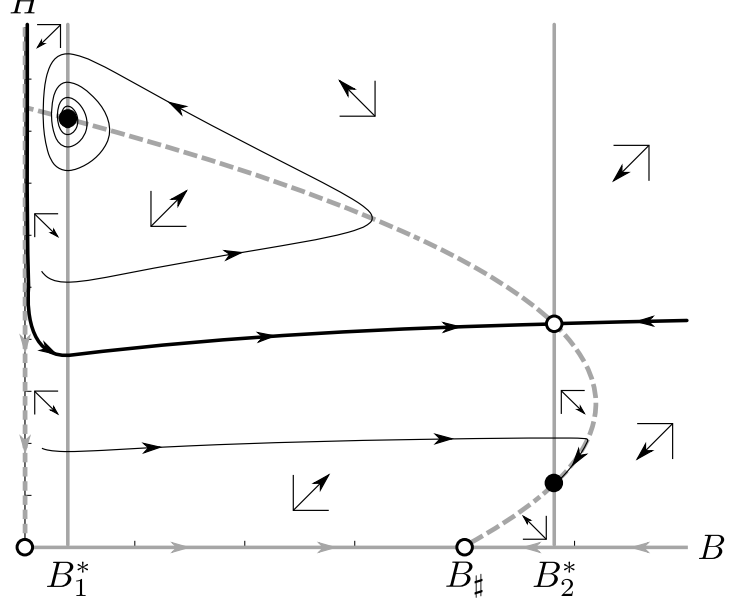

Figure 6: Typical phase plane diagrams of case (iii) (stable overcompensation and herbivore-free equilibria, panel (a)) and case (v) (stable overcompensation and undercompensation equilibria, panel (b)). Panel (a) is computed from the Direct compensation version of model (8) with parameters as in Figure 4, with $\gamma=8$ and $\mu=0.185$ identified by the asterisk. It is also qualitatively representative of the case (iii) phase plane obtained from the Direct compensation and Resistance model. Panel (b) is computed from the Direct compensation and Resistance version of model (8) in the case where $B_{\sharp}>B_{m}$, with parameters as in Figure 4 and $\gamma=8$ and $\mu=0.1596$ identified by the asterisk on panel (b). Stable equilibria are represented with black dots, saddle points with white circles. The $\dot{H}=0$ nullclines are represented with plain grey lines and $\dot{B}=0$ with dashed grey lines; the qualitative orientation of the trajectories in the phase plane is represented accordingly with arrows. Black thick curves are separatrix curves which determine the respective basins of attraction of the stable equilibria. Black thin curves are typical model trajectories. 


\begin{tabular}{|c|c|c|}
\hline Functions & & Hypotheses \\
\hline$r_{B}(H)$ & in & $\begin{array}{l}\quad r_{B}(0)>0 \\
\text { creasing for small } H \\
H<H_{m} \text { with } H_{m} \in[0,+\infty]\end{array}$ \\
\hline$\delta(B)$ & & $\begin{array}{l}0 \leq \delta(0)<r_{B}(0) \\
\text { increasing } \\
\delta(B)>\sup _{H \in \mathbb{R}_{+}} r_{B}(H)\end{array}$ \\
\hline$\phi(B)$ & \multirow{2}{*}{\multicolumn{2}{|c|}{\begin{tabular}{c|c} 
Direct compensation & $\begin{array}{c}\text { Direct compensation and Resistance } \\
\alpha(0)=0\end{array}$ \\
nonnegative & $\begin{array}{c}\text { nonnegative } \\
\text { increasing }\end{array}$ \\
$\mu<\lim _{B \rightarrow+\infty} \alpha(B)$ & increasing for $B<B_{m}$ and \\
decreasing for $B>B_{m}$ with $B_{m} \in \mathbb{R}_{*}^{-}$ & $\mu<\alpha\left(B_{m}\right)$
\end{tabular}}} \\
\hline$\alpha(B)$ & & \\
\hline
\end{tabular}

Table 1: Hypotheses on the different functions used in system (1)

\begin{tabular}{|c|c|c|c|c|}
\hline \multicolumn{2}{|c|}{ Existence conditions } & Equilibria & Stable biological equilibria & Cases \\
\hline \multicolumn{2}{|l|}{$\mathcal{C}_{a}<1$} & $\begin{array}{c}\left(B_{\sharp}, 0\right) \text { unstable } \\
\left(B^{*}, H_{2}^{*}\right) \mathrm{LAS}^{\dagger}\end{array}$ & Undercompensation & (i) \\
\hline \multirow{3}{*}{$\mathcal{C}_{a}>1$} & $\mathcal{C}_{s}<1$ & $\left(B_{\sharp}, 0\right) \mathrm{LAS}$ & Herbivore-free & (ii) \\
\hline & \multirow[b]{2}{*}{$\mathcal{C}_{s}>1$} & $\left(B_{\sharp}, 0\right) \mathrm{LAS}$ & Herbivore-free & (ii) \\
\hline & & $\begin{array}{c}\left(B_{\sharp}, 0\right) \text { LAS } \\
\left(B^{*}, H_{1}^{*}\right) \text { unstable } \\
\left(B^{*}, H_{2}^{*}\right) \text { LAS }^{\dagger}\end{array}$ & $\begin{array}{c}\text { Herbivore-free } \\
\text { Overcompensation }\end{array}$ & (iii) \\
\hline
\end{tabular}

Table 2: Different Equilibria and their stability/unstability properties. $\dagger$ signs highlight the equilibria for which stability is linked to the condition $\delta^{\prime}\left(B^{*}\right)+\phi^{\prime}\left(B^{*}\right) H_{2}^{*}>0$, otherwise instability occurs through Hopf bifurcation. This condition is always verified if $\phi$ is taken constant. Three distinct cases have been identified. When $\mathcal{C}_{s}>1$ and $\mathcal{C}_{a}>1$, the knowledge of $\mathcal{C}_{s}$ and $\mathcal{C}_{a}$ is not sufficient to determine the number of equilibria without any further knowledge on the system. The cases numbering corresponds to the one of Figure 2 


\begin{tabular}{|c|c|c|c|c|}
\hline \multicolumn{2}{|c|}{ Existence conditions } & Equilibria & Stable biological equilibria & Cases \\
\hline \multicolumn{2}{|l|}{$1>\mathcal{C}_{a 2}>\mathcal{C}_{a 1}$} & $\begin{array}{c}\left(B_{\sharp}, 0\right) \text { LAS } \\
\left(B_{1}^{*}, H_{12}^{*}\right) \text { LAS }^{\dagger} \\
\left(B_{2}^{*}, H_{22}^{*}\right) \text { unstable }\end{array}$ & $\begin{array}{c}\text { Herbivore-free } \\
\text { Undercompensation }\end{array}$ & (iv) \\
\hline \multirow{3}{*}{$\mathcal{C}_{a 2}>1>\mathcal{C}_{a 1}$} & $1>\mathcal{C}_{s 2}$ & $\begin{array}{l}\left(B_{\sharp}, 0\right) \text { unstable } \\
\left(B_{1}^{*}, H_{12}^{*}\right) \mathrm{LAS}^{\dagger}\end{array}$ & Undercompensation & (i) \\
\hline & \multirow[b]{2}{*}{$\mathcal{C}_{s 2}>1$} & $\begin{array}{l}\left(B_{\sharp}, 0\right) \text { unstable } \\
\left(B_{1}^{*}, H_{12}^{*}\right) \mathrm{LAS}^{\dagger}\end{array}$ & Undercompensation & (i) \\
\hline & & $\begin{array}{c}\left(B_{\sharp}, 0\right) \text { unstable } \\
\left(B_{1}^{*}, H_{12}^{*}\right) \mathrm{LAS}^{\dagger} \\
\left(B_{2}^{*}, H_{21}^{*}\right) \mathrm{LAS}^{\dagger} \\
\left(B_{2}^{*}, H_{22}^{*}\right) \text { unstable }\end{array}$ & $\begin{array}{l}\text { Undercompensation } \\
\text { Overcompensation }\end{array}$ & $(\mathrm{v})$ \\
\hline \multirow{7}{*}{$\mathcal{C}_{a 2}>\mathcal{C}_{a 1}>1$} & $1>\mathcal{C}_{s 2} \geq \mathcal{C}_{s 1}$ & $\left(B_{\sharp}, 0\right)$ LAS & Herbivore-free & (ii) \\
\hline & & $\left(B_{\sharp}, 0\right) \mathrm{LAS}$ & Herbivore-free & (ii) \\
\hline & $\mathcal{C}_{s 2}>1>\mathcal{C}_{s 1}$ & $\begin{array}{c}\left(B_{\sharp}, 0\right) \text { LAS } \\
\left(B_{2}^{*}, H_{21}^{*}\right) \mathrm{LAS}^{\dagger} \\
\left(B_{2}^{*}, H_{22}^{*}\right) \text { unstable }\end{array}$ & $\begin{array}{c}\text { Herbivore-free } \\
\text { Overcompensation }\end{array}$ & (iii-bis) \\
\hline & \multirow{4}{*}{$\mathcal{C}_{s 2} \geq \mathcal{C}_{s 1}>1$} & $\left(B_{\sharp}, 0\right)$ LAS & Herbivore-free & (ii) \\
\hline & & $\begin{array}{c}\left(B_{\sharp}, 0\right) \text { LAS } \\
\left(B_{1}^{*}, H_{11}^{*}\right) \text { unstable } \\
\left(B_{1}^{*}, H_{12}^{*}\right) \mathrm{LAS}^{\dagger}\end{array}$ & $\begin{array}{c}\text { Herbivore-free } \\
\text { Overcompensation }\end{array}$ & (iii) \\
\hline & & $\begin{array}{c}\left(B_{\sharp}, 0\right) \text { LAS } \\
\left(B_{2}^{*}, H_{21}^{*}\right) \text { LAS }^{\dagger} \\
\left(B_{2}^{*}, H_{22}^{*}\right) \text { unstable }\end{array}$ & $\begin{array}{c}\text { Herbivore-free } \\
\text { Overcompensation }\end{array}$ & (iii-bis) \\
\hline & & $\begin{array}{c}\left(B_{\sharp}, 0\right) \text { LAS } \\
\left(B_{1}^{*}, H_{11}^{*}\right) \text { unstable } \\
\left(B_{1}^{*}, H_{12}^{*}\right) \text { LAS }^{\dagger} \\
\left(B_{2}^{*}, H_{21}^{*}\right) \text { LAS }^{\dagger} \\
\left(B_{2}^{*}, H_{22}^{*}\right) \text { unstable }\end{array}$ & $\begin{array}{c}\text { Herbivore-free } \\
\text { Two overcompensations }\end{array}$ & (vi) \\
\hline
\end{tabular}

Table 3: Different equilibria and their stability/instability properties when both $B_{1}^{*}$ and $B_{2}^{*}$ exist. $\dagger$ signs highlight the equilibria for which stability is linked to the condition $\delta^{\prime}\left(B_{i}^{*}\right)+\phi^{\prime}\left(B_{i}^{*}\right) H_{i j}^{*}>0$, otherwise instability occurs through Hopf bifurcation. This condition is always verified if $\phi$ is taken constant. Seven distinct cases have been identified corresponding to six different biological configurations. When $\mathcal{C}_{s 2}>1$ and $\mathcal{C}_{a 2}>1$, the knowledge of $\mathcal{C}_{s i}$ and $\mathcal{C}_{a i}$ is not sufficient to determine the number of equilibria without any further knowledge on the system. The cases numbering corresponds to the one of Figure 3 